\title{
Simulation of reactions involving charged particles in hypersonic rarefied flows
}

\author{
Erin D. Farbar* and Iain D. Boyd ${ }^{\dagger}$ \\ Department of Aerospace Engineering, University of Michigan, Ann Arbor, MI 48109
}

\begin{abstract}
During vehicle reentry into the Earth's atmosphere from space, the flow field energy is sufficient to produce ionizing reactions. The weakly ionized plasma around the vehicle can interfere with vehicle communications, and in some cases can have an important effect on the rate of heat transfer to the capsule surface. In the present study, the modeling of reactions involving charged species is considered. Axisymmetric simulations of hypersonic flow about the forebody of the FIRE II reentry vehicle using the Direct Simulation Monte Carlo (DSMC) method are presented, with the flight conditions of the vehicle at $85 \mathrm{~km}$ altitude used as the test case. The baseline flow field solution is computed using the commonly used Total Collision Energy (TCE) chemistry model to simulate reactions involving charged particles. The standard chemistry model is then replaced with both measured and computed cross section data for the simulation of electron impact ionization, associative ionization and dissociation of $\mathrm{N}_{2}$ by electron impact. The results obtained using the improved model are compared to those obtained with the baseline model, and the changes in the flow field variables are systematically examined. The use of reaction cross section data for modeling electron impact dissociation has a large effect on the computed flow field composition. The use of the reaction cross section data for associative ionization and electron impact ionization has a limited effect on the charged species concentrations in the flow field. In order to use the reaction cross section data for associative ionization reactions in a DSMC calculation that does not model the electronic mode explicitly, a means of determining the electronic state of the reactants is required.
\end{abstract}

\section{Nomenclature}

d diameter in $\mathrm{m}$

$\mathrm{F}_{i} \quad$ fraction of particles occupying state i

$\mathrm{g}_{i} \quad$ degeneracy of energy level i

k Boltzmann constant

Kn Knudsen number

$\mathrm{n}$ number density in $\mathrm{m}^{-3}$

$\mathrm{p}$ pressure in $\mathrm{N} / \mathrm{m}^{2}$

$\mathrm{P}$ probability of an event

$\mathrm{R}_{n}$ radius of capsule forebody

$\mathrm{T}$ temperature in $\mathrm{K}$

$\mathrm{U}$ velocity in $\mathrm{km} / \mathrm{s}$

$\rho \quad$ density in $\mathrm{kg} / \mathrm{m}^{3}$

$\sigma \quad$ cross section

$\omega \quad$ parameter in VHS model

$\varepsilon \quad$ mode energy

$\nu \quad$ vibrational level

\footnotetext{
*Graduate Student, Student Member AIAA. Email: efarbar@umich.edu

${ }^{\dagger}$ Professor, Associate Fellow AIAA. Email: iainboyd@umich.edu
} 


\section{Subscripts}

e electron value

$\mathrm{r}$ rotational value

ref reference value

$\mathrm{t}$ translational value

thres threshold value

v vibrational value

$\infty$ freestream value

\section{Introduction}

When vehicles enter a planetary atmosphere at super-orbital velocities, a weakly ionized plasma may be created. This plasma causes communications blackout, changes the structure of the flow field surrounding the vehicle and affects the rate of convective and radiative heat transfer to the vehicle surface. For these reasons, when computing high energy, rarefied entry trajectories using the Direct Simulation Monte Carlo (DSMC) method, it is necessary to incorporate models for the interactions of ionized particles into the algorithm.

One of the most widely used chemistry models in the DSMC algorithm is the Total Collision Energy model. ${ }^{1}$ In this model, the probability of two particles reacting once they have been selected for a collision is derived such that the overall reaction rate at equilibrium corresponds to an experimentally determined or recommended reaction rate coefficient of Arrhenius form. The nature of this model is such that the shape of the reaction cross section is determined solely by the constraint that the reaction rate at equilibrium match the measured reaction rate. The reaction cross section derived using this model may not compare well with experimental reaction cross section data determined as a function of particle collision energy and internal state. It was demonstrated in Refs. 2 and 3 that the addition of ionization chemistry to the simulation of the FIRE II experiment resulted in changes in the predicted flow field and in the rate of heat transfer predicted at the capsule surface. Additionally, an accurate prediction of the electron translational temperature and the concentrations of charged species in the flow field are required for the computation of the radiative component of the heat flux, which can be significant at some flight conditions of hypersonic vehicles. For example, during a Titan aerocapture utilizing a ballute-type geometry, the radiative portion of the vehicle heating is likely to be a substantial portion of the total heat flux. Additionally, due to the nature of the materials that could be used in an aerocapture system, the tolerable heat flux will likely be much lower than that of an Earth entry system, necessitating a smaller margin of error in the aerothermal analysis. For these reasons it is important to develop the capability to accurately model reactions involving charged species in rarefied flows.

The DSMC method has previously been used to analyze weakly ionized flow fields. For example, the DSMC technique has been applied in the past to analyze the flow about the Aero-assisted Orbital Transfer Vehicle and a generic blunt vehicle at velocities of up to $10 \mathrm{~km} / \mathrm{s},{ }^{4,5}$ the flow field about the FIRE II vehicle at a velocity of $11.3 \mathrm{~km} / \mathrm{s}^{2,3}$ and the flow field about the more recent Stardust reentry vehicle ${ }^{6,7}$ at a freestream velocity of $12.6 \mathrm{~km} / \mathrm{s}$. Unfortunately the amount of experimental data obtained from these vehicles is very limited. All of this previously published work utilized chemistry models that were composed entirely of Arrhenius rate coefficients for the important reactions in the eleven species air model. These rate coefficients were obtained from data obtained at experimental conditions that are, in some cases, not representative of the environment experienced by a reentry vehicle. ${ }^{8}$ As changes to the chemistry model can have large effects on the predicted flow field composition, ${ }^{9}$ it is important to explore the limitations of this chemistry model.

The purpose of this paper is to examine the effect of using cross section data for reactions involving charged species on the analysis of a low-density hypersonic flow field in which strong non-equilibrium effects are present. To this end, reaction cross section data has been gathered for reactions in eleven species air and used to form a new chemistry model for electron impact ionization of nitrogen and oxygen, associative ionization of nitrogen and oxygen, and electron impact dissociation of molecular nitrogen. Reaction probabilities obtained using the cross section data are compared to those computed using the TCE model. Next, DSMC results for the flow field about the FIRE II vehicle at $85 \mathrm{~km}$ are computed using the new cross section data and systematically compared to the results obtained using the standard TCE model. Differences between the predictions for flow field variables such as the concentrations of charged species, the mode temperatures, and the heat flux to the capsule surface are identified. Conclusions are drawn as to the significance of the 
changes observed in the predicted flow field structure when the cross section data is used in lieu of the TCE model for the reactions examined in this work.

\section{The Fire II flight experiment}

Project FIRE was an Apollo era experiment to measure the radiative and convective heating during atmospheric entry at lunar return speeds. ${ }^{10}$ The FIRE II reentry vehicle consisted of three phenolic-asbestos heat shields sandwiched between beryllium calorimeters. The first two heat shields and calorimeter packages were designed to be ejected after the onset of melting, yielded heating data free of the effects of ablation and three separate data gathering periods. Calorimeter plugs and radiometers were located at various positions on the heat shields. ${ }^{10}$ Figure 1 is an image of the FIRE II vehicle. The free stream conditions for the simulation are given in Table 1. The freestream Knudsen number is based on the capsule forebody diameter.

\section{Details of the baseline DSMC model}

Electrons have a mass that is five orders of magnitude lower than that of the heavier air species, and their addition to the flow field necessitates modifications to the DSMC method. If the electrons possess the same translational temperature as the other species, their thermal velocities will be more than two orders of magnitude higher. However, the transport of the electrons will be limited by the electrostatic attraction of the ions and electrons. This phenomenon can be modeled using the assumption of ambipolar diffusion, which requires that ions and electrons move at the same average rate to maintain charge neutrality on scales larger than the Debye length scale. The collision frequency is proportional to the collision cross section and the relative velocity of the colliding pair. Due to their high thermal velocities, if one particle of the pair is an electron this rate will be very high, thus requiring a much smaller simulation time step as compared to simulations performed without electrons. Simulations of weakly ionized flow fields thus in general become much more computationally expensive.

In this work, the effect of the electric field is simulated simply by invoking the assumption of ambipolar diffusion and requiring the electrons to move at the average ion velocity in a given cell. ${ }^{11}$ Models for rotational $^{12}$ and vibrational ${ }^{13}$ relaxation during inelastic collisions are included, along with the standard neutral chemistry set and an additional 31 reaction baseline chemistry set involving charged particles and including both associative and direct ionizing reactions. ${ }^{6}$ The complete chemistry set is given in Ref. 3 . The reaction rates are implemented within the DSMC framework using the Total Collision Energy (TCE) model, and the Vibrationally-Favored Dissociation (VFD) model ${ }^{14}$ is implemented for the dissociation of molecular nitrogen and oxygen utilizing favoring parameters of 2.0 and 0.5 , respectively. The set of Variable Hard Sphere (VHS) parameters ${ }^{15}$ used to model particle interactions is given in Table 2. The molecular properties of the ions are set equal to the properties of the corresponding neutral particles.

The vehicle wall is assumed to be fully catalytic to ions and electrons but not catalytic to atoms. The grid spacing in the direction of flow gradients is less than the local mean free path, and the computational time step is less than the local heavy particle collision time everywhere in the domain. The collision routine is subcycled within each overall simulation time step to accurately simulate collisions of electrons and heavy particles, and the particles are moved with a timestep corresponding to the collision time of heavy particles. Electron-electron collisions are not simulated as these are very frequent and serve solely to thermalize the electron distribution function; it is assumed that collisions of electrons with heavy species are sufficient to do so.

The TCE model has been used in previous work to model the dissociation of nitrogen by electron impact, the ionization of atoms by electron impact and the associative ionization of atomic nitrogen and oxygen. These reaction mechanisms are given in Equations 1,2,3, and 4 and the reaction rate coefficients are given in Table 3. Translational temperature contours in the FIRE II flowfield at the $85 \mathrm{~km}$ flight condition obtained in earlier work ${ }^{3}$ using the TCE model and these rate coefficients are shown in Fig. 2. The flow field is in a significant state of thermal nonequilibrium as indicated by the highly disparate temperatures of the various energy modes along the stagnation streamline shown in Fig. 3. Additionally, there is a significant degree of ionization occurring in the flow field, as evidenced in Fig. 4, which shows contours of electron number density for the same flight condition. An electron number density greater than $1 \times 10^{15} \mathrm{~m}^{-3}$ is sufficient to interfere with radio communication to the vehicle, ${ }^{16}$ and the results at this flight condition are well in excess of that. 


$$
\begin{aligned}
& N_{2}+e \rightarrow N+N+e \\
& N+e \rightarrow N^{+}+e+e \\
& O+e \rightarrow O^{+}+e+e \\
& N+O \leftrightarrow N O^{+}+e
\end{aligned}
$$

\section{Details of the improved chemistry model}

In order to accurately predict the level of ionization, the chemical reactions that produce charged species must be modeled accurately. The accurate prediction of the energy content of the flow field requires a good knowledge of the cross sections for dissociation, and in ionized air flow the electron impact dissociation of nitrogen is an important reaction mechanism. ${ }^{3}$ In this work, an improved model for these reactions is presented that involves using calculated and measured cross section data as opposed to average reaction rate coefficients to determine a reaction event. For all reaction mechanisms discussed, Equation 5 is used to cast the cross section data into an expression for reaction probability suitable for use with the DSMC algorithm.

$$
P_{\text {react }}=\frac{\sigma_{\text {react }}}{\sigma_{\text {total }}}
$$

\section{IV.A. Modeling of electron impact dissociation}

The reaction mechanism, as given by Equation 6, proceeds via predissociation of an electronically excited $\mathrm{N}_{2}^{*}$ state. $^{17}$

$$
N_{2}+e \rightarrow N_{2}^{*}+e \rightarrow N+N+e
$$

There exists experimental cross section data due to Cosby ${ }^{17}$ obtained using a crossed beam experiment and two different nitrogen ion sources that were neutralized by near-reasonant charge transfer. This experiment yielded cross section measurements for electron-impact energies between 10 and $200 \mathrm{eV}$. The vibrational and electronic state populations of the $\mathrm{N}_{2}$ were not measured but it was deduced that in the hollow cathode ion source, more than $90 \%$ of the $\mathrm{N}_{2}^{+}$population was in the ground electronic and vibrational state. It was estimated that in the $\mathrm{N}_{2}$ beam produced using the electron impact ion source no more than $24 \%$ of the nitrogen was vibrationally excited, and again that it was in the ground electronic level. The analysis of the translational energy distributions of the product particles did indeed indicate that the predissociation of bound $\mathrm{N}_{2}^{*}$ states makes the dominant contribution to the electron impact dissociation of $\mathrm{N}_{2}$, rather than the excitation of the molecule to, and subsequent dissociation from, a dissociative continuum. The comparison of the cross section data obtained with the two different ion sources did not yield any systematic differences, despite their different vibrational populations.

Based on these results, it is assumed in the following analysis that the cross section data due to Cosby are absolute cross sections, in that they include all product states of the nitrogen atoms and electron over the tested energy range, and that the cross sections for dissociation are equal for all vibrational and rotational levels of the $\mathrm{N}_{2}$ molecule in the ground electronic state. Because in general the probability of a termolecular collision is extremely low in the rarefied gas regime, recombination is not considered. It is assumed in the reaction mechanics of the DSMC code that the colliding nitrogen molecule is in the ground electronic state, and the resulting nitrogen atoms are also in their ground states yielding a threshold energy of of $9.75 \mathrm{eV} .{ }^{17}$ It is unclear whether the internal energy of the $\mathrm{N}_{2}$ molecule should contribute to the reaction process, given the predissociation mechanism discussed above. In the results presented here it is assumed that the internal energy of the nitrogen molecule does not contribute to the threshold energy.

Figure 5 shows the reaction probability computed using the measured cross section data and that computed using the TCE model with the rate coefficient given in Table 3. For consistency when comparing the reaction probability produced by the TCE model to that computed with the cross section data, the internal energy of the $\mathrm{N}_{2}$ molecule was not included when calculating the reaction probability using the TCE model. 
However, when the TCE model is used in practice, it is typically assumed that all of the internal energy of the $\mathrm{N}_{2}$ molecule contributes to the reaction. This is the case for the simulation results presented in this work. It is therefore instructive to compare the reaction rate coefficient produced by the cross section data to that used in the TCE model. Figure 6 shows the variation of both rate coefficients with temperature. It is clear that the rate coefficient produced by the Cosby data is lower than that used in the TCE model at all temperatures. That rate coefficient was originally estimated based on the requirement that existing radiation data from shock tubes be reproduced. ${ }^{8}$

\section{IV.B. Modeling of electron impact ionization}

Experimental and theoretical cross section data for the electron impact ionization of oxygen and nitrogen were compiled by Bell et al. ${ }^{18}$ Figures 7 and 8 compare the reaction probabilities obtained using the TCE model and the rate coefficients given in Table 3 as a function of collision energy to those computed using the recommended cross sections from Ref. 18. The Arrhenius rate coefficients used in the TCE model correspond to those measured for the rate of ionization of nitrogen and oxygen from their first excited states. ${ }^{19}$ The data and curve-fits for the total cross sections used to compute the ionization probability were documented in Ref. 3.

For both reaction mechanisms it is clear that in the energy range of interest, the TCE model yields reaction probabilities for individual collisions that are higher than those determined theoretically or experimentally. The reaction rate coefficients obtained from these data sets are compared to those used in the TCE model in Figures 9 and 10. The rate coefficients used in the TCE model are larger those obtained from the cross section data at all temperatures.

\section{IV.C. Modeling of associative ionization}

Due to their low threshold energies, associative ionization (AI) reactions play an important role in determining the level of ionization and structure of the flow field at the flight conditions considered in this work. There exists very little experimental or computational data in the literature regarding the associative ionization reactions in air. The associative ionization reaction of $\mathrm{N}+\mathrm{O}$ has the lowest energy threshold, so this is the reaction that is analyzed in this work. Although the associative ionization of $\mathrm{N}+\mathrm{N}$ is the most important associative ionization mechanism for the given flight condition, cross section data for this reaction could not be located in the literature for use in this analysis.

\section{IV.C.1. Forward reaction, $\mathrm{N}+\mathrm{O} \rightarrow \mathrm{NO}^{+}+e$}

Padellec ${ }^{20}$ computed the partial reaction cross sections in the vicinity of the threshold energies for the associative ionization of $\mathrm{N}+\mathrm{O}$ to form $\mathrm{NO}^{+}+\mathrm{e}(\nu=0$, ground electronic state). The AI process involves the capture of two neutral atoms into an electronically bound, discrete state $\left(\mathrm{NO}^{*}\right)$, and subsequent transition to the unbound $\mathrm{NO}^{+}+$e state with which it is degenerate. ${ }^{21}$ The cross sections were computed by invoking the principle of microscopic reversibility ${ }^{22}$ and are a function of the electronic state of the colliding atoms. The three sets of electronic states of the colliding atoms considered by Padellec that yielded useful data sets and the corresponding threshold energies for each reaction are listed below.

$$
\begin{aligned}
& E 1: N\left({ }^{4} S\right)+O\left({ }^{3} P\right), E_{\text {thres }}=2.77 \mathrm{eV} \\
& E 2: N\left({ }^{4} S\right)+O\left({ }^{1} D\right), E_{\text {thres }}=0.80 \mathrm{eV} \\
& E 3: N\left({ }^{2} D\right)+O\left({ }^{3} P\right), E_{\text {thres }}=0.38 \mathrm{eV}
\end{aligned}
$$

Additionally, Ringer and Gentry ${ }^{23}$ measured the absolute cross section for the AI of $\mathrm{N}\left({ }^{2} \mathrm{D}\right)+\mathrm{O}\left({ }^{3} \mathrm{P}\right)$. In order to cast these sets of cross section data in a form useful in the DSMC algorithm, one has to have some information about the electronic state of the reactants, as well as the shape and magnitude of the cross sections reported by Padellec away from the threshold energy. The latter requires including the additional partial cross sections for ionization resulting in vibrationally and possibly electronically excited states of the $\mathrm{NO}^{+}$ion. Ringer and Gentry were able to place an upper limit on the magnitude of the cross section corresponding to threshold E1 of $15 \%$ of that corresponding to E3. ${ }^{23}$ Consistent with this limit, it is assumed in this work that the absolute cross section for ionization from the first two reactant states is of the same 
shape and relative magnitude as that measured by Ringer and Gentry for the third energy threshold. The cross sections constructed for each reactant state are shown in Figure 11, along with the original data sets. Also shown on that figure is the cross section computed using the TCE chemistry model with the rate coefficient given in Table 3. The rate coefficient used in the TCE model was deduced from measured rates for the dissociative recombination reaction of $\mathrm{NO}^{+}+\mathrm{e}$ and the appropriate equilibrium constant. ${ }^{8}$

\section{IV.C.2. Reverse reaction, $\mathrm{NO}^{+}+e \rightarrow \mathrm{N}+\mathrm{O}$}

In DSMC, particles are first selected for a collision and then each pair is tested for subsequent energy exchange and reactions using computed probabilities for each possible event. Due to the long range nature of the Coulomb interaction, the cross section for collisions between charged particles is much larger than that for collisions of charged particles with neutrals and between neutral particles. To employ such a high collision rate in a DSMC solution of an entire vehicle flow field, as is typically done in smaller Particle in Cell (PIC) type simulations for example, would be prohibitively expensive. Additionally, due to the relatively low concentration of charged species in these types of flow fields, the effect of such a high collision rate on the flow parameters of interest would likely be very small. Instead, the reaction cross sections for the dissociative recombination (DR) reaction are inserted directly into the collision selection algorithm in the DSMC code, and the corresponding reaction probability is then set equal to unity in the reaction selection algorithm. This means that all collisions of $\mathrm{NO}^{+}+\mathrm{e}$ will react, and none will involve only elastic or inelastic energy transfer. Although from a microscopic point of view this is unphysical behavior, it should produce the correct level of ionization in the flow field without requiring the computation of very large numbers of collisions between charged particles.

Vejby-Christensen et al. ${ }^{24}$ measured the total cross sections for $\mathrm{DR}$ of $\mathrm{NO}^{+}+\mathrm{e}$ in the vibrational and electronic ground state, as well as the branching ratios for the product $\mathrm{N}+\mathrm{O}$ atoms. This data was used by Padellec in the computation of the forward AI reaction cross sections. The Vejby-Christensen data is compared to the cross section computed using the TCE chemistry model and the rate coefficient listed in Table 3 in Fig. 12. Also shown on this plot is the curve-fit used to implement the cross section data in the code. Note that the set of data produced using the TCE chemistry model utilizes a reaction probability that is a function of total collision energy. In order to use the Vejby-Christensen cross section data in a DSMC calculation, some assumption has to be made about the cross sections for DR from vibrational states other than $\nu=0$. In this work it is assumed that the reaction cross sections from states with $\nu>0$ are equal to those with $\nu=0$.

\section{IV.C.3. Equilibrium reservoir calculation}

An equilibrium reservoir simulation is performed to calculate the total forward and reverse reaction rate coefficients, determine the equilibrium constant and compare to that tabulated by Park. ${ }^{25}$ In order to do so, the electronic state populations of the nitrogen and oxygen atoms are calculated using the appropriate Boltzmann distribution given in Equation 8, at the electronic temperature of the flow (in this case the reservoir temperature). The joint probability of occurrence for each set of electronic states listed in Equation 7 is then formed, referred to from here on as an electronic co-state. In doing so, it is assumed that the cross sections for reaction from electronic co-states at higher energies than those given by the E3 threshold are equal to those for the E3 threshold. Figure 13 shows the distribution of each co-state given in Equation 7 as a function of reservoir temperature. The quantity labelled " $1-\mathrm{P}(\mathrm{E} 1)-\mathrm{P}(\mathrm{E} 2)$ " on this figure is the probability that is used to identify collisions that proceed using the E3 threshold data. The quantity labelled "P(E1) $+\mathrm{P}(\mathrm{E} 2)+\mathrm{P}(\mathrm{E} 3)$ " corresponds to the fraction of possible electronic co-states of the $\mathrm{N}$ and $\mathrm{O}$ atoms that are accounted for at a given electronic temperature. For example, at a temperature of $20000 \mathrm{~K}$, approximately $75 \%$ of the possible electronic co-states are accounted for using this method. When a nitrogen atom is selected to collide with an oxygen atom in the simulation, a random number is generated and used to determine the appropriate co-state of the pair. The probability of a reaction is then computed using the appropriate cross section data for that electronic co-state.

$$
F_{i}=\frac{g_{i} e^{-\epsilon_{i} / k T_{e}}}{\sum g_{i} e^{-\epsilon_{i} / k T_{e}}}
$$

Figure 14 shows the reaction probabilities obtained from the equilibrium reservoir simulation for each of the three sets of forward cross section data when electronic excitation is not considered, that is when each 
set of data is applied in a separate simulation. Also shown in red on this figure is the reaction probability obtained when the electronic states of the $\mathrm{N}$ and $\mathrm{O}$ atoms are modeled according to the method outlined above. Figure 15 shows the corresponding total forward reaction rate coefficient. Also shown on these figures are the results obtained using the TCE model. At temperatures greater than approximately $10000 \mathrm{~K}$, the reaction probability and forward rate coefficient calculated using the cross section data is greater than that predicted by the TCE model.

Figure 16 shows the results for the equilibrium constant obtained from the equilibrium reservoir simulation. The agreement between the equilibrium constant used by the TCE model and that given by Park is fairly good at temperatures less than $30000 \mathrm{~K}$. This is expected since the equilibrium constant used in the TCE model was fit over this finite temperature range to be cast in a form usable in the model. Further discussion of this point is found in Ref. 9. The equilibrium constant computed using the cross section data agrees with that given by Park over a smaller temperature range, but agrees much better at lower reservoir temperatures. A major source of the disagreement between the equilibrium constant computed using the AI and DR cross section data and the Park value is due to the fact that AI cross sections have been inferred for the higher electronic states of $\mathrm{N}$ and $\mathrm{O}$, and the $\mathrm{DR}$ cross sections have been inferred for the higher vibrational states of $\mathrm{NO}^{+}$. At an equilibrium temperature of $15000 \mathrm{~K}, 10 \%$ of the atoms are in electronic states higher than those accounted for in the Padellec data and that percentage increases rapidly above $15000 \mathrm{~K}$. The cross sections for ionization from these states are set equal to those for the E3 threshold. At an equilibrium temperature of $15000 \mathrm{~K}$, almost all $\mathrm{NO}^{+}$ions are in vibrational states higher than $\nu=0$, and the cross sections for dissociative recombination from these states are are set equal to those measured for the $\nu=0$ state.

\section{IV.C.4. Application in the FIRE II vehicle simulation}

The electronic mode temperature is assumed equal to the electron translational temperature in the DSMC simulation due to the high efficiency of energy exchange between free and bound electrons. The electron translational temperature is typically between $10000 \mathrm{~K}$ and $20000 \mathrm{~K}$ in the simulations of the FIRE II 1631 s flight condition, within the region of good agreement between the equilibrium constant computed from the data and that computed from the Park model, as shown in Fig. 16. Thus, the AI cross section data can be employed reliably to compute the flow field if some means of determining the electronic states of the colliding atoms is implemented. Of course one option is to directly model the electronic excitation of the atoms in the DSMC algorithm, ${ }^{2}$ or alternatively to employ a quasi-steady state approximation to compute the population of the electronic states as is typically done in codes designed to compute flow field radiation in an uncoupled manner. ${ }^{26,27}$ In order to first get a sense as to how much of an effect the use of the AI cross section data in lieu of the TCE model will have on the computed flow field, in this work the electronic energy levels of both the nitrogen and oxygen atoms are assumed to be populated according to the equilibrium distribution at the local electron translational temperature of the flow field. The procedure for reaction selection described in Section IV.C.3 is then used.

\section{Results and Discussion}

\section{V.A. Electron impact dissociation of $\mathrm{N}_{2}$}

Figure 17 shows a comparison of the mode temperatures along the stagnation streamline computed using the TCE chemistry model and those computed using the Cosby cross section data. Due to the reduced reaction probability associated with the cross section data, there are fewer dissociation events and the internal mode temperatures and the electron translational temperature are increased. Figure 18 shows that the mole fractions of charged species along the stagnation line have significantly increased due to the high energy electrons now present in the flow. Specifically, the mole fractions of $\mathrm{O}^{+}$and $\mathrm{N}^{+}$have increased significantly, presumably due to the increased importance of the electron impact ionization reactions in the presence of the higher energy electrons. The maximum local degree of ionization in the flow field has increased by over $50 \%$ to slightly greater than 0.03 in the post-shock region. Lastly, Fig. 19 shows that the heat flux along the capsule surface is slightly increased with the use of the Cosby data, due to the reduced level of nitrogen dissociation in the flow field. A data point from one of the calorimeters on the FIRE II heat shield is also shown in this figure. The addition of the cross section data results in slightly worse agreement with the experimental data, however this is hardly a conclusive result because there are many other sources of 
uncertainty in the physical and chemical data used in these types of simulations.

Although the inclusion of the Cosby reaction cross section data has a relatively small effect on the convective heat transfer shown in Fig. 19, it plays a large role in the computation of the radiative portion of the heat flux. This is due to the increase of the electron translational temperature in the flow field, as the electronic temperature is assumed equal to the electron translational temperature for the purposes of calculating the electronic state populations of the heavy particles. An uncoupled calculation of the total radiative heat flux to the capsule surface using the HARA code ${ }^{26}$ yields a value of $8 \mathrm{~W} / \mathrm{cm}^{2}$ using the flow field results computed using the TCE model as input, while this value increases to $24 \mathrm{~W} / \mathrm{cm}^{2}$ for the flow field computed using the Cosby data. This may have important implications for flow fields where the radiative heating is a more significant portion of the total heat flux.

\section{V.B. Electron impact ionization of atomic species}

The next set of results employs both the data for electron impact dissociation, as well as that for electron impact ionization. The addition of the former produced higher electron temperatures, which resulted in the electron impact reactions playing a larger role in determining the flow field character when the TCE model was used. Figure 20 shows the mole fractions of charged species along the stagnation streamline of the flow are reduced to very near the values obtained using the baseline chemistry set. A reduction in the level of ionization is expected as the ionization cross sections from the ground electronic level given by Bell et al. are lower than those predicted using the equilibrium rates given in the baseline chemistry set. The mode temperatures along the stagnation streamline are shown in Fig. 21. The mode temperatures are almost completely unchanged from the previous result computed using only the data for e- $\mathrm{N}_{2}$ dissociation. This is likely due to the fact that the ionization energies used in the TCE model with the Wilson rates for $\mathrm{N}+\mathrm{e}$ and $\mathrm{O}+$ e ionization are substantially lower than those used with the cross section data, as the particles are assumed to ionize from the first excited state in the TCE model. Thus, even though fewer electron impact ionization events are taking place, the net energy loss in the bulk flow region is approximately the same. The heat transfer at the probe surface is largely unchanged from the values obtained using only the $\mathrm{N}_{2}+\mathrm{e}$ dissociation data, as expected.

\section{V.C. Associative ionization of $\mathrm{N}+\mathrm{O}$}

Figure 22 shows the mole fractions of charged species along the stagnation streamline computed using the TCE model and those computed using the cross section data for the associative ionization of $\mathrm{N}+\mathrm{O}$. As discussed previously, in order to utilize this data it is assumed that electronic state populations of the atoms are in equilibrium at the instantaneous local electron temperature of a given cell. As shown in Fig. 15 , the reaction rate coefficient predicted by the data is higher than that predicted by the TCE model for temperatures greater than $10000 \mathrm{~K}$. Figure 3 shows that once an appreciable degree of ionization has occurred in the flow field, the electron temperature rises to approximately $14000 \mathrm{~K}$. Thus, the AI reaction of $\mathrm{N}+\mathrm{O}$ should play a more prominent role in the flow field when the cross section data is employed, and this is precisely what is shown in Fig. 22. The concentration of the product $\mathrm{NO}^{+}$has increased in the region of the flow where $\mathrm{T}_{e}>10000 \mathrm{~K}$, while the concentrations of $\mathrm{N}^{+}$and $\mathrm{O}^{+}$have decreased slightly in this region, likely because more of the energetic atoms are being consumed by the AI reaction. The net effect is that the overall the degree of ionization is not appreciably changed from the baseline solution. The mode temperatures and convective heat flux to the vehicle surface are not affected by the addition of the $\mathrm{N}+\mathrm{O}$ AI data.

\section{Conclusions}

The use of cross section data for DSMC computations of Earth reentry flows presents difficulties as, in general, data is not available for all combinations of reactant states typically present in high temperature air. This requires assumptions to be made about the missing data, and those assumptions cannot be validated. Additionally, adding reaction cross section data to a DSMC code that does not include a coupled model for the electronic mode presents the additional problem of determining the electronic state of the colliding particles in order to apply the appropriate cross section for reaction.

With appropriate assumptions, reaction cross section data has been systematically implemented into the DSMC algorithm for the electron impact dissociation of nitrogen, electron impact ionization of nitrogen and 
oxygen, and associative ionization of nitrogen and oxygen. This data replaces the rate coefficients previously used for the analysis of Earth entry, that were obtained from limited experimental data and fit to the form necessary for use with the TCE chemistry model.

The addition of cross section data for the electron impact dissociation of nitrogen molecules had a substantial affect on the computed flow field about the FIRE II vehicle at $1631 \mathrm{~s}$. The peak degree of ionization increased by approximately $50 \%$ to a maximum value of 0.03 in the post-shock region. The convective heat flux along the capsule surface increased by approximately $7 \%$. Although the radiative portion of the vehicle heating at this flight condition is low, it was tripled to $24 \mathrm{~W} / \mathrm{cm}^{2}$ with the use of this cross section data.

When the electron impact ionization data was added to the chemistry model while retaining the electron impact dissociation data, the peak degree of ionization was reduced to $2 \%$, approximately the value obtained using the original chemistry set. The mode temperatures and convective heat transfer were unaffected.

The addition of only the cross section data for associative ionization of $\mathrm{N}+\mathrm{O}$ to the simulation resulted in an almost $100 \%$ increase in the peak concentration of $\mathrm{NO}^{+}$in the flow field relative to the results obtained using the original chemistry set. The peak concentrations of $\mathrm{O}^{+}$and $\mathrm{N}^{+}$decreased slightly and the other flow field results were unaffected.

Due to the substantial effect the addition of the $\mathrm{N}_{2}$ - e dissociation data had on the prediction of the radiative component of the heat flux, it is recommended that it be added, along with the electron impact ionization data, to chemistry models used for analysis of energetic entry missions such as Titan aerocapture with ballute-type geometries. At those types of flight conditions, the radiative heating is likely to contribute substantially to the total heat flux even in the rarefied flow regime.

\section{Acknowledgments}

The authors gratefully acknowledge the financial support provided by NASA grant NCC3-989.

\section{References}

${ }^{1}$ Bird, G. A., Molecular Gas Dynamics and the Direct Simulation of Gas Flows, Oxford Science Publications, 1994.

${ }^{2}$ Taylor, J. C., Carlson, A. B., and Hassan, H. A., "Monte Carlo Simulation of Radiating Re-entry Flows," Journal of Thermophysics and Heat Transfer, Vol. 8, No. 3, 1994, pp. 478-485.

${ }^{3}$ Farbar, E. D. and Boyd, I. D., "Simulation of Fire II Reentry Flow Using the Direct Simulation Monte Carlo Method," 40th AIAA Thermophysics Conference, 2008, AIAA 2008-4103.

${ }^{4}$ Bird, G. A., "Nonequilibrium Radiation During Re-entry at 10 km/s." AIAA 22nd Thermophysics Conference, 1987, AIAA-87-1543

${ }^{5}$ Boyd, I. D. and Gokcen, T., "Compuation of Axisymmetric and Ionized Hypersonic Flows Using Particle and Continuum Method," AIAA Journal, Vol. 32, 1987, pp. 1828-1835.

${ }^{6}$ Boyd, I. D., Trumble, K., and Wright, M. J., "Nonequilibrium Particle and Continuum Analyses of Stardust Entry for Near-Continuum Conditions," 38th AIAA Aerospace Sciences Meeting and Exhibit, 2007, AIAA-2007-4543.

${ }^{7}$ Ozawa, T., Nompelis, I., Levin, D. A., Barnhardt, M., and Candler, G. V., "DSMC-CFD Comparison of a High Altitude, Extreme-Mach Number Reentry Flow," 46th AIAA Aerospace Sciences Meeting and Exhibit, 2008, AIAA-2008-1216.

${ }^{8}$ Park, C., Nonequilibrium Hypersonic Aerothermodynamics, John Wiley and Sons, 1990.

${ }^{9}$ Boyd, I. D., "Modelling backward chemical rate processes in the direct simulation Monte Carlo method," Physics of Fluids, Vol. 19, 2007, Article 126103.

${ }^{10}$ Cornette, E. S., "Forebody Temperatures and Calorimeter Heating Rates Measured During Project Fire II Reentry at 11.35 Kilometers Per Second," Tech. Rep. NASA TM X-1305, Langley Research Center, 1966.

${ }^{11}$ Boyd, I. D., "Monte Carlo Simluation of Nonequilibrium Flow in a Low-power Hydrogen Arcjet," Physics of Fluids, Vol. 9, No. 10, 1997, pp. 4575-4584.

${ }^{12}$ Boyd, I. D., "Rotational-translational Energy Transfer in Rarefied Nonequilibrium Flows," Physics of Fluids, Vol. 2, No. 3, 1989, pp. 447-452.

${ }^{13}$ Boyd, I. D., "Analysis of Vibrational-translational Energy Transfer Using the Direct Simulation Monte Carlo Method," Physics of Fluids, Vol. 3, No. 7, 1991, pp. 1785-1791.

${ }^{14}$ Haas, B. L. and Boyd, I. D., "Models for Direct Monte Carlo Simulation of Coupled Vibration-Dissociation," Physics of Fluids, Vol. 5, No. 2, 1993, pp. 478-489.

${ }^{15}$ Bird, G. A., "Monte Carlo Simulation in an Engineering Context," Rarefied Gas Dynamics, edited by S. S. Fisher, Vol. 74 of Progress in Astronautics and Aeronautics, AIAA, New York, 1981, pp. 239-255.

${ }^{16}$ Keidar, M., Kim, M., and Boyd, I. D., "Electromagnetic Reduction of Plasma Density During Atmospheric Reentry and Hypersonic Flights," The Journal of Spacecraft and Rockets, Vol. 45, No. 3, 2008, pp. 445-453.

${ }^{17}$ Cosby, P. C., "Electron impact dissociation of nitrogen," Journal of Chemical Physics, Vol. 98, No. 12, 1993, pp. 95449553. 
${ }^{18}$ Bell, K. L., Gilbody, H. B., Hughes, J. G., Kingston, A. E., and Smith, F. J., "Recommended Data on the Electron Impact Ionization of Light Atoms and Ions," Journal of Physical and Chemical Reference Data, Vol. 12, No. 4, 1983, pp. 891-917.

${ }^{19}$ Wilson, J., "Ionization rate of air behind high-speed shock waves," The Physics of Fluids, Vol. 9, No. 10, 1966, pp. 19131921.

${ }^{20}$ Padellec, A. L., "Partial Near Threshold Cross Sections for the Associative Ionization to form $\mathrm{CO}^{+}, \mathrm{NO}^{+}$and $\mathrm{O}_{2}^{+}$," Physica Scripta, Vol. 71, 2005, pp. 621-626.

${ }^{21}$ Nielson, S. E. and Dahler, J. S., "Endoergic chemi-ionization in N-O collisions," Journal of Chemical Physics, Vol. 71, No. 4, 1979, pp. 1910-1925.

${ }^{22}$ Mahan, B. H., "Microscopic Reversibility and Detailed Balance: an analysis," Journal of Chemical Education, Vol. 52, No. 5, 1975, pp. 299-302.

${ }^{23}$ Ringer, G. and Gentry, W. R., "A merged molecular beam study of the endoergic associative ionization reaction $\mathrm{N}\left({ }^{2} \mathrm{D}\right)+\mathrm{O}\left({ }^{3} \mathrm{P}\right) \rightarrow \mathrm{NO}^{+}+\mathrm{e}^{-}, "$ Journal of Chemical Physics, Vol. 71, No. 4, 1979, pp. 1902-1909.

${ }^{24}$ Vejby-Christensen, L., Kella, D., Pedersen, H. B., and Andersen, L. H., "Dissociative recombination of $\mathrm{NO}^{+}$," Physical Review A, Vol. 57, No. 5, 1998, pp. 3627-3634.

${ }^{25}$ Park, C., Jaffe, R. L., and Partridge, H., "Chemical-Kinetic Parameters of Hyperbolic Earth Entry," Journal of Thermophysics and Heat Transfer, Vol. 15, No. 1, 2001, pp. 76-90.

${ }^{26}$ Johnston, C. O., "Nonequilibrium Shock-Layer Radiative Heating for Earth and Titan Entry," Ph.D. Dissertation, Virginia Tech, November 2006.

${ }^{27}$ Whiting, E., Park, C., Liu, Y., Arnold, J. O., and Paterson, J. A., "NEQAIR96, Nonequilibrium and Equilibrium Radiative Transport and Spectra Program: User's Manual," Tech. Rep. NASA RP-1389, 1996. 
Table 1: Flow conditions for Project FIRE II at $1631 \mathrm{~s}$

\begin{tabular}{lc}
\hline \hline Property & $1631 \mathrm{~s}$ \\
\hline Altitude & $84.6 \mathrm{~km}$ \\
$\rho_{\infty}$ & $9.15 \times 10^{-6} \mathrm{~kg} / \mathrm{m}^{3}$ \\
$\mathrm{U}_{\infty}$ & $11.37 \mathrm{~km} / \mathrm{s}$ \\
$\mathrm{T}_{\infty}$ & $212 \mathrm{~K}$ \\
$\mathrm{~T}_{\text {wall }}$ & $460 \mathrm{~K}$ \\
$\mathrm{R}_{n}$ & $0.9347 \mathrm{~m}$ \\
$\mathrm{Kn}_{\infty}$ & 0.01 \\
\hline \hline
\end{tabular}

Table 2: Parameters used in the VHS model.

\begin{tabular}{lc}
\hline \hline Parameter & Value \\
\hline$\omega$ & 0.20 \\
$\mathrm{~T}_{r e f}$ & $288 \mathrm{~K}$ \\
$\mathrm{~d}_{N_{2}}$ & $4.07 \times 10^{-10} \mathrm{~m}$ \\
$\mathrm{~d}_{O_{2}}$ & $3.96 \times 10^{-10} \mathrm{~m}$ \\
$\mathrm{~d}_{N O}$ & $4.00 \times 10^{-10} \mathrm{~m}$ \\
$\mathrm{~d}_{N}$ & $3.00 \times 10^{-10} \mathrm{~m}$ \\
$\mathrm{~d}_{O}$ & $3.00 \times 10^{-10} \mathrm{~m}$ \\
$\mathrm{~d}_{e}$ & $1.00 \times 10^{-10} \mathrm{~m}$ \\
\hline \hline
\end{tabular}

Table 3: Reaction rate coefficients used in the TCE chemistry model, $\mathrm{m}^{3} /$ molecule/s.

\begin{tabular}{lc}
\hline \hline Reaction & Rate Coefficient \\
\hline $\mathrm{N}_{2}+\mathrm{E}^{-} \rightarrow \mathrm{N}+\mathrm{N}+\mathrm{E}^{-}$ & $4.980 \times 10^{-6} \mathrm{~T}^{-1.6} \exp (-113200 / \mathrm{T})$ \\
$\mathrm{N}+\mathrm{E}^{-} \rightarrow \mathrm{N}^{+}+2 \mathrm{E}^{-}$ & $8.434 \times 10^{-14} \exp (-121000 / \mathrm{T})$ \\
$\mathrm{O}+\mathrm{E}^{-} \rightarrow \mathrm{O}^{+}+2 \mathrm{E}^{-}$ & $1.054 \times 10^{-14} \exp (-106200 / \mathrm{T})$ \\
$\mathrm{N}+\mathrm{O} \rightarrow \mathrm{NO}^{+}+\mathrm{e}$ & $8.766 \times 10^{-18} \exp (-32006 / \mathrm{T})$ \\
$\mathrm{NO}^{+}+\mathrm{e} \rightarrow \mathrm{N}+\mathrm{O}$ & $1.321 \times 10^{-9} \mathrm{~T}^{-1.187}$ \\
\hline \hline
\end{tabular}

11 of 25 


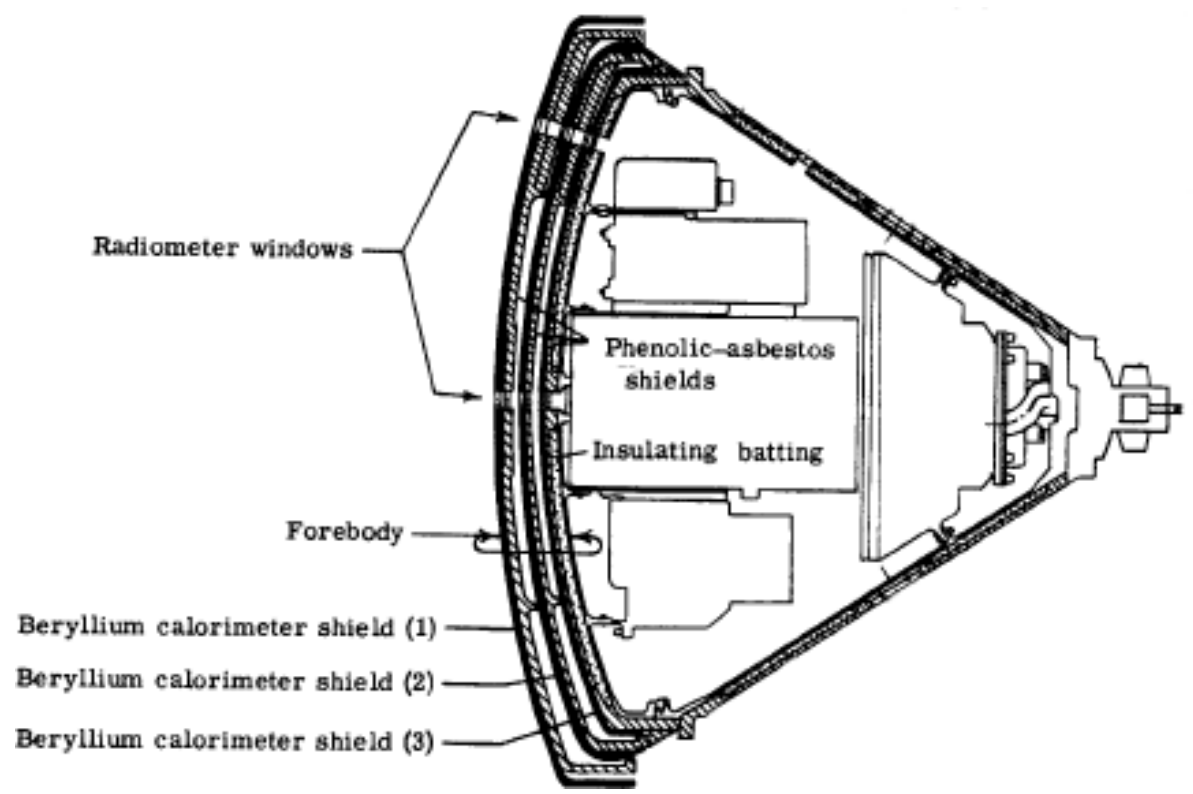

Figure 1: Image of FIRE II vehicle. ${ }^{10}$ 


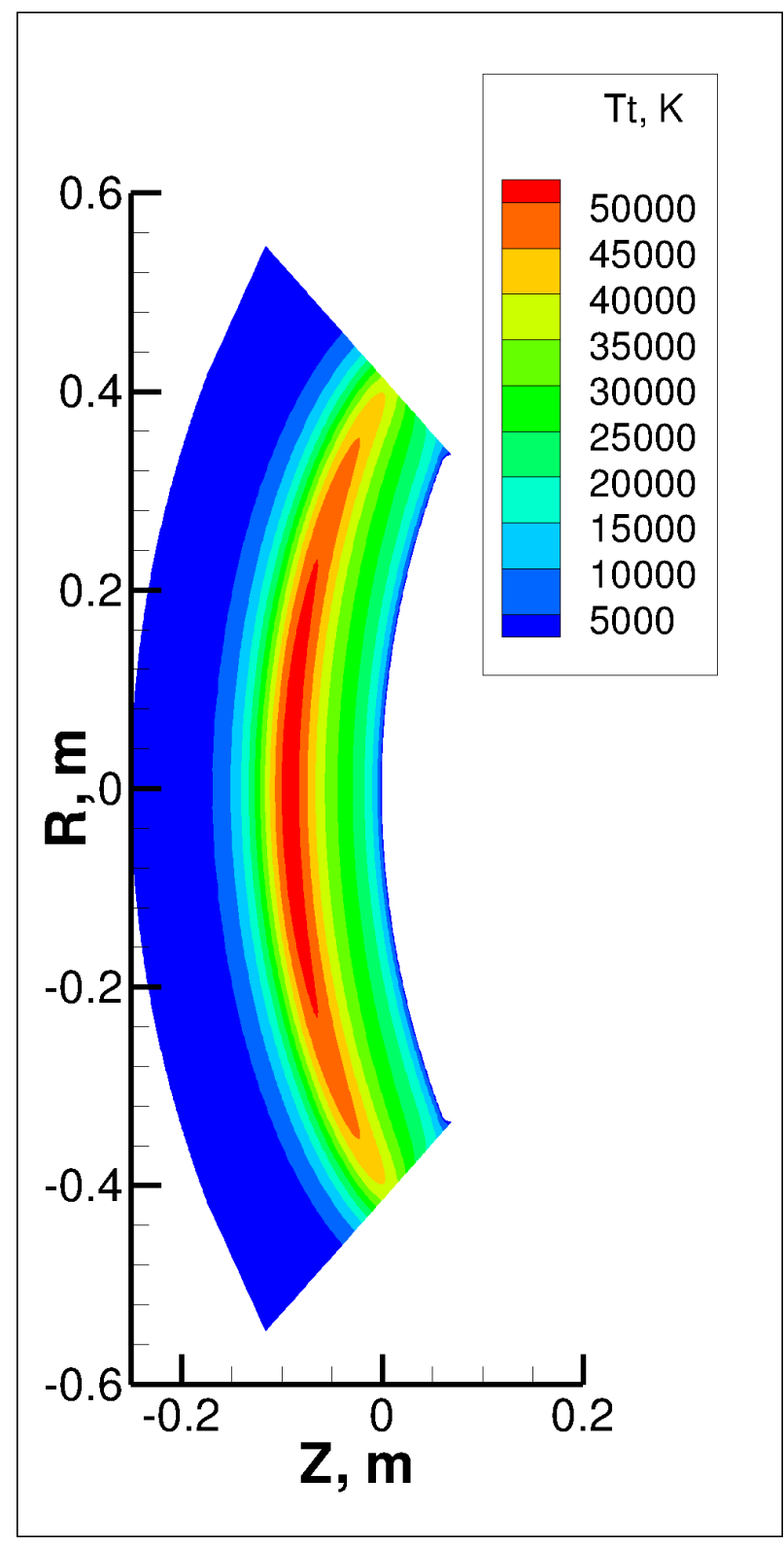

Figure 2: Contours of translational temperature for FIRE II at the $85 \mathrm{~km}$ flight condition. 


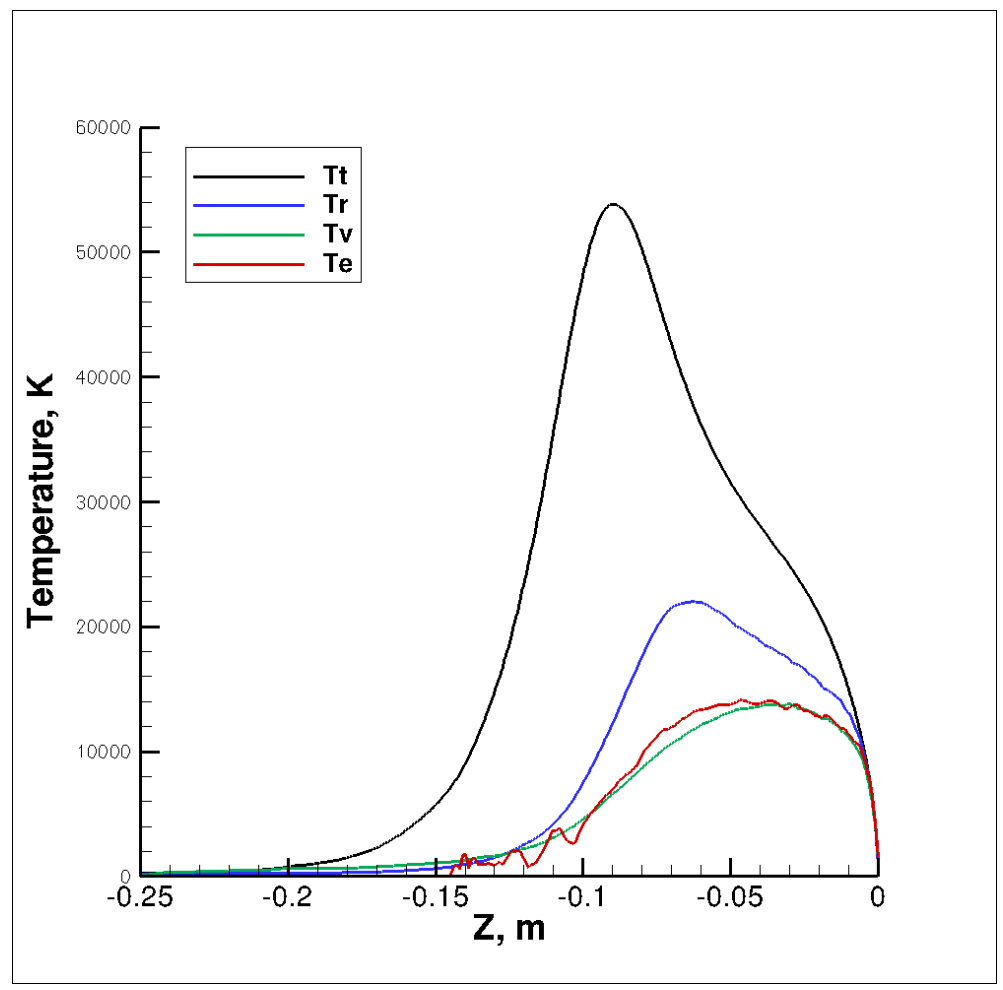

Figure 3: Temperature profiles along the stagnation streamline for FIRE II at the $85 \mathrm{~km}$ flight condition. 


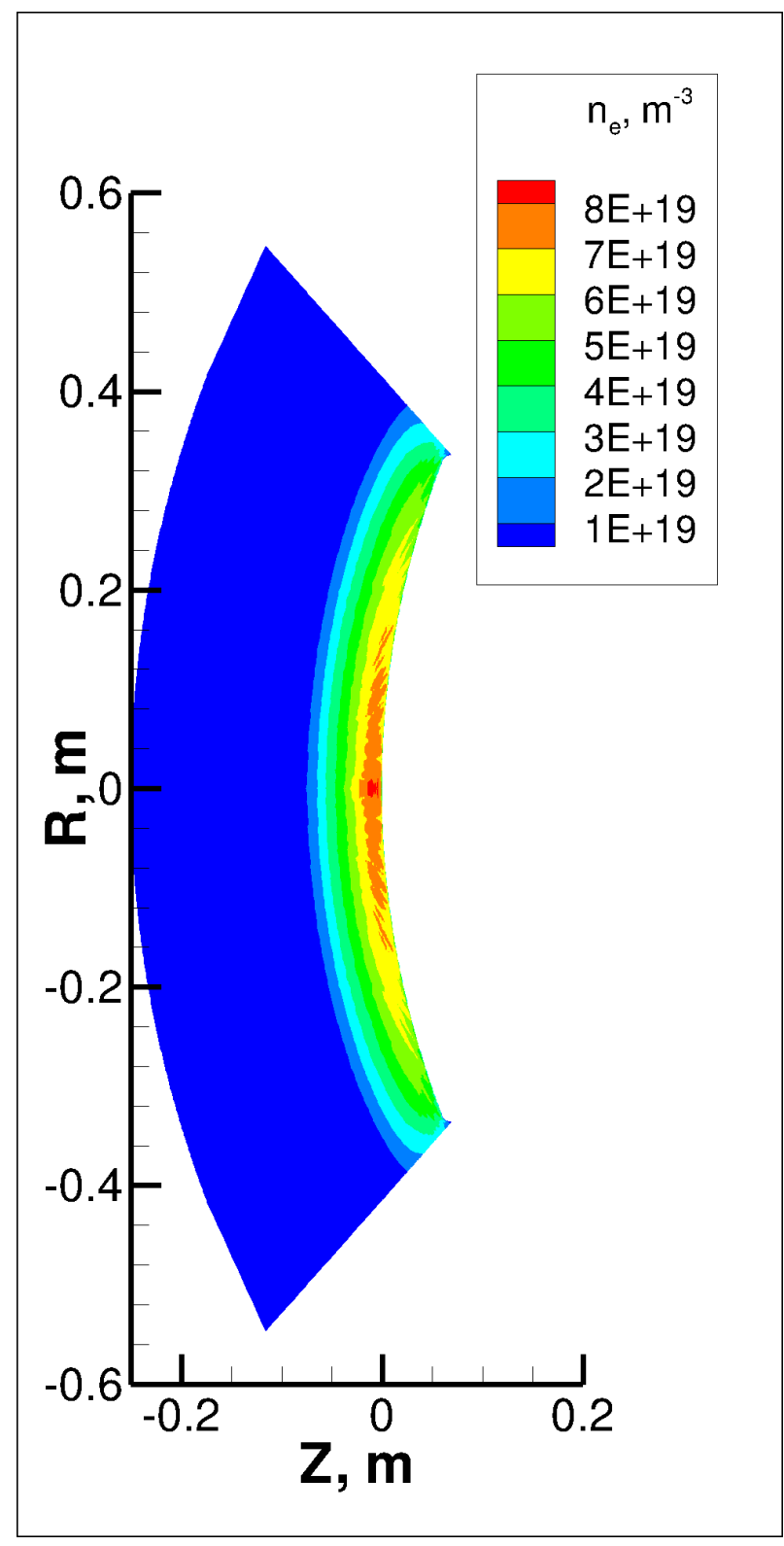

Figure 4: Contours of electron number density for FIRE II at the $85 \mathrm{~km}$ flight condition. 


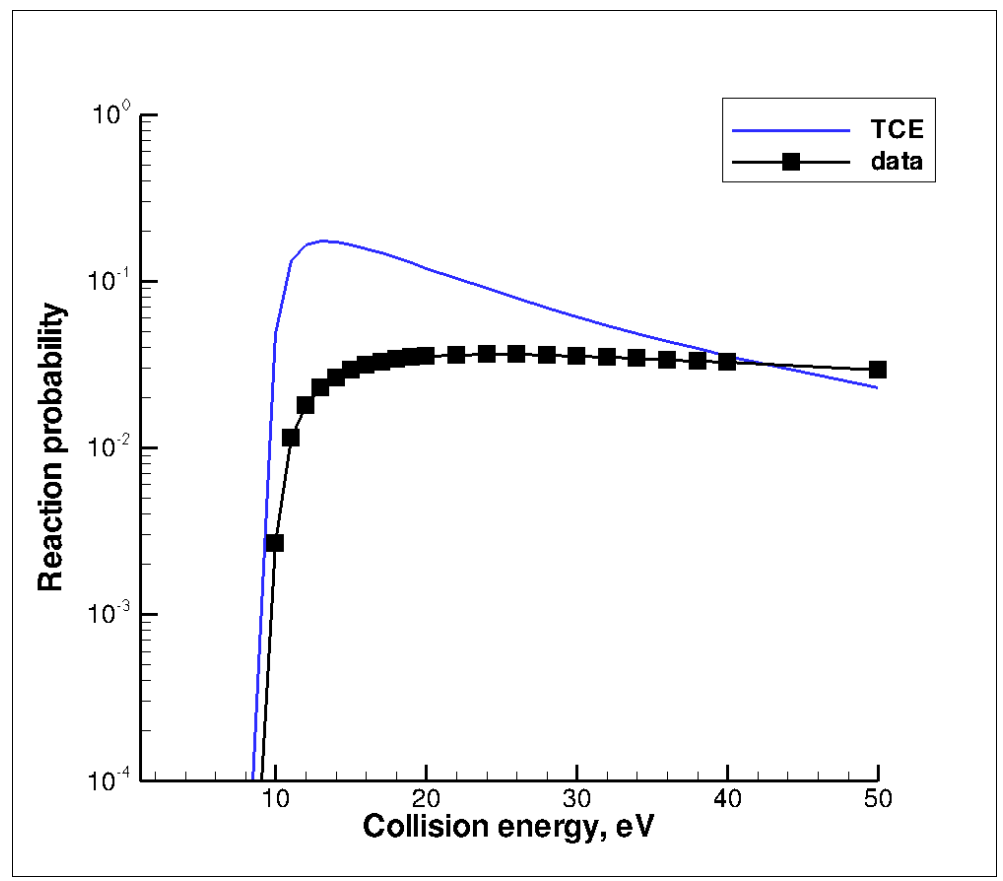

Figure 5: Reaction probability for the electron impact dissociation of nitrogen given by Eqn. (1) computed using both the TCE model and the cross section data from Ref. 17. 


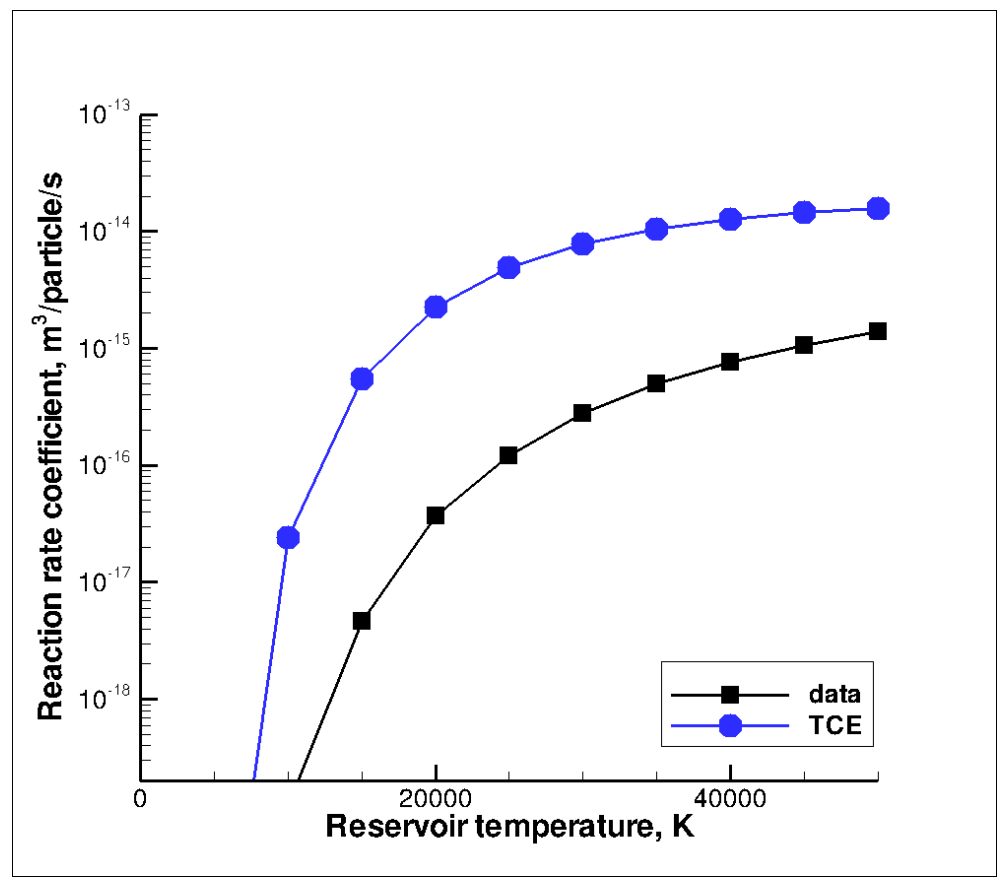

Figure 6: Reaction rate coefficient for the electron impact dissociation of nitrogen given by Eqn. (1).

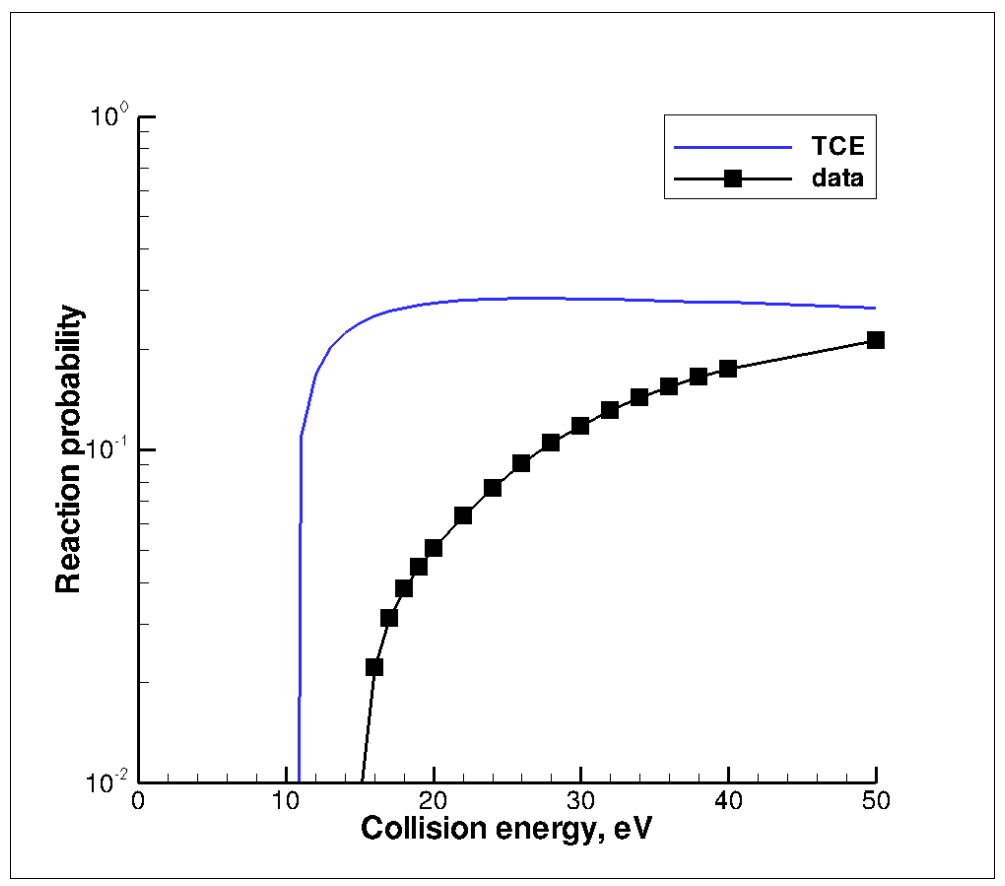

Figure 7: Reaction probability for the electron impact ionization of nitrogen given by Eqn. (2) computed using both the TCE model and the cross section data from Ref. 18. 


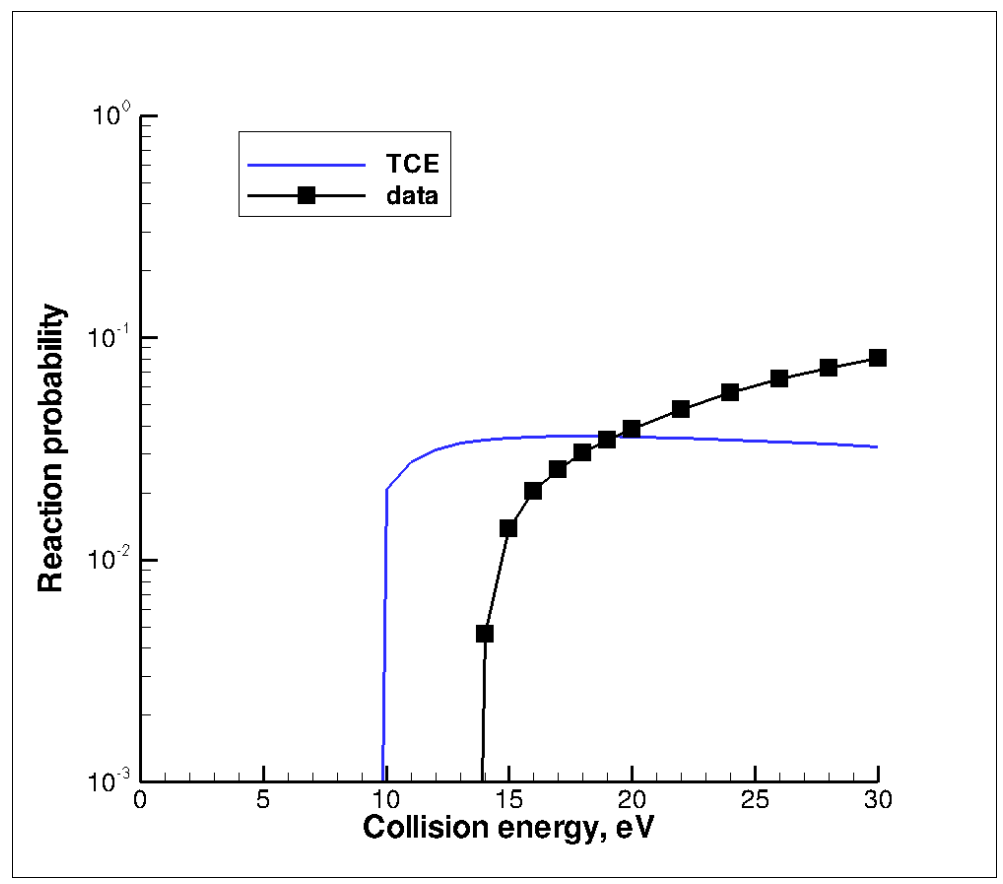

Figure 8: Reaction probability for the electron impact ionization of oxygen given by Eqn. (3) computed using both the TCE model and the cross section data from Ref. 18.

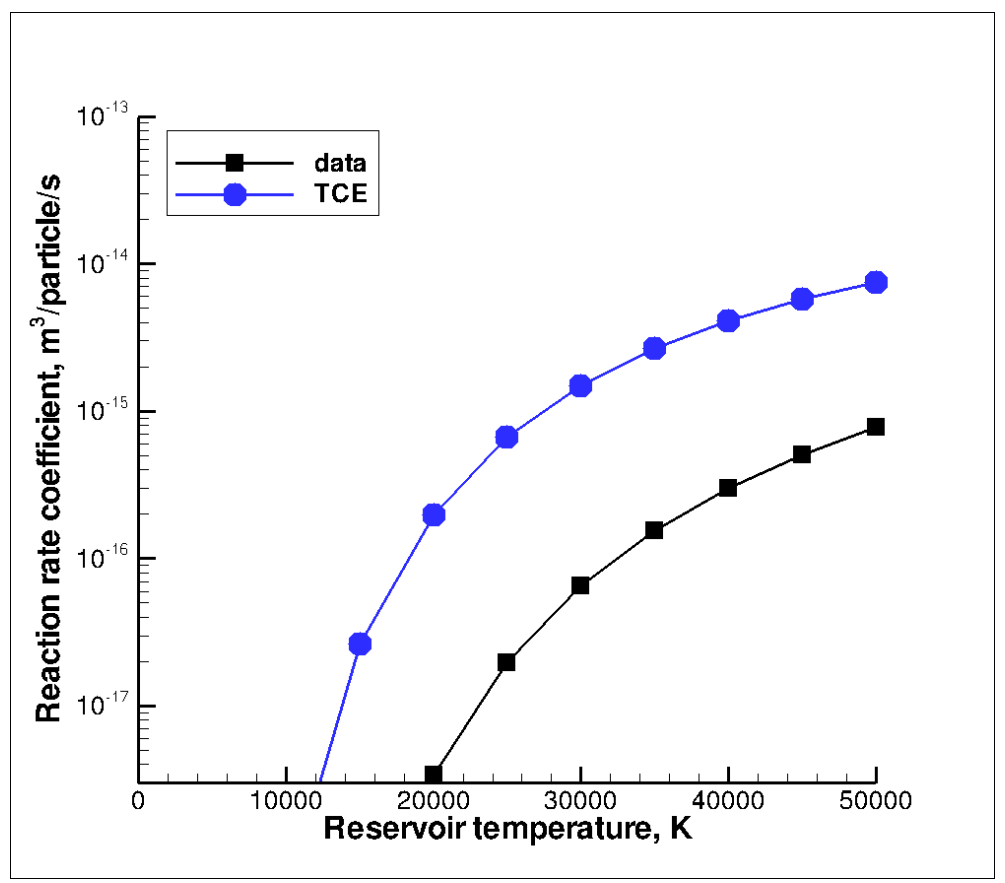

Figure 9: Reaction rate coefficient for the electron impact ionization of nitrogen given by Eqn. (2). 


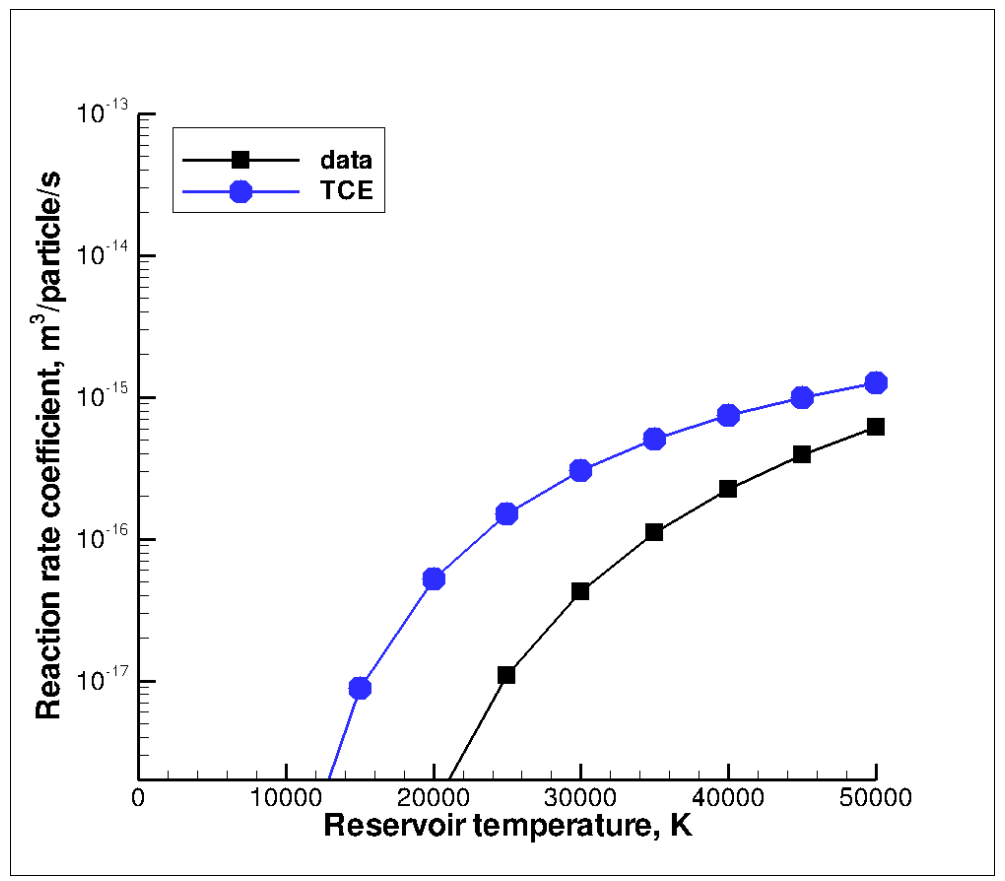

Figure 10: Reaction rate coefficient for the electron impact ionization of oxygen given by Eqn. (3).

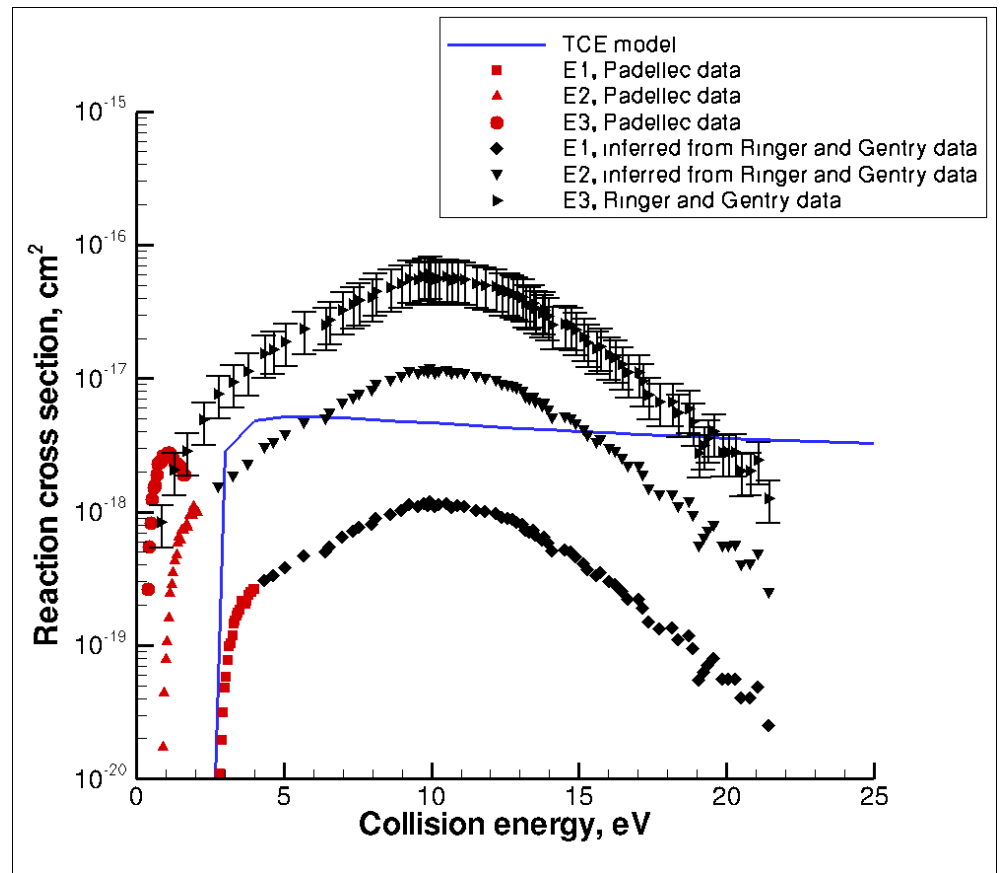

Figure 11: Cross sections for the associative ionization of $\mathrm{N}+\mathrm{O}$ given by Eqn. (4). 


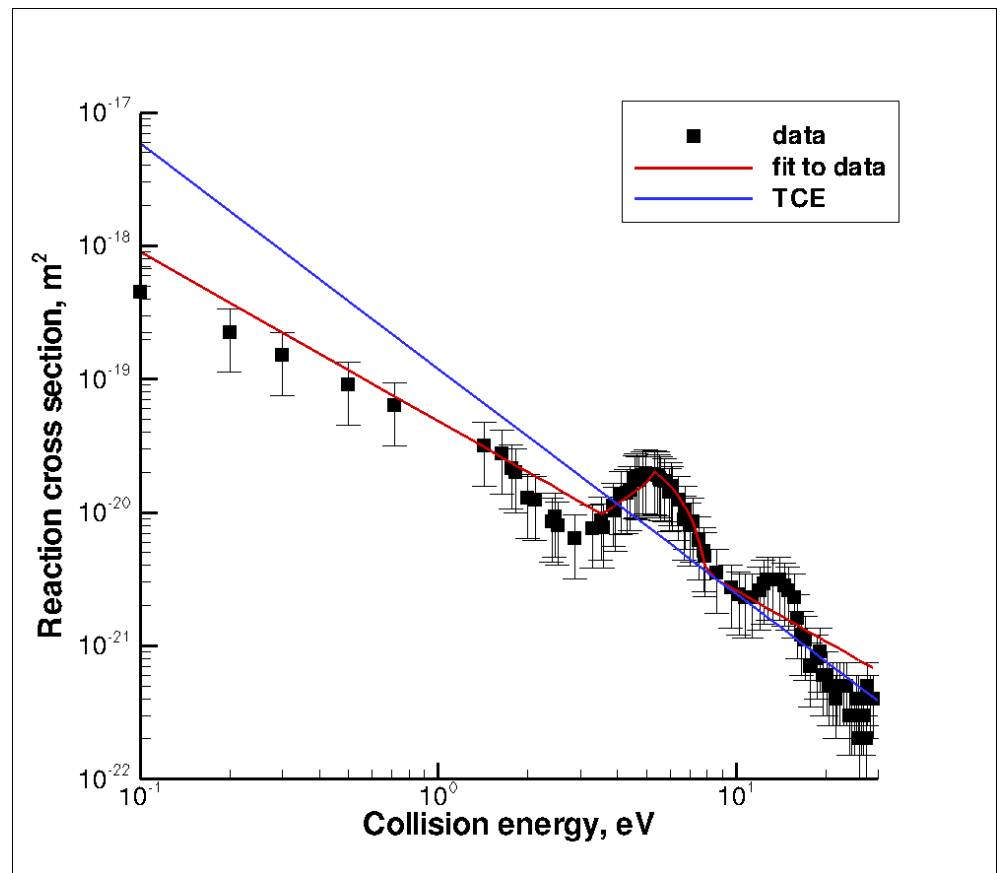

Figure 12: Cross sections for the dissociative recombination of $\mathrm{NO}^{+}+$e given by Eqn. (4).

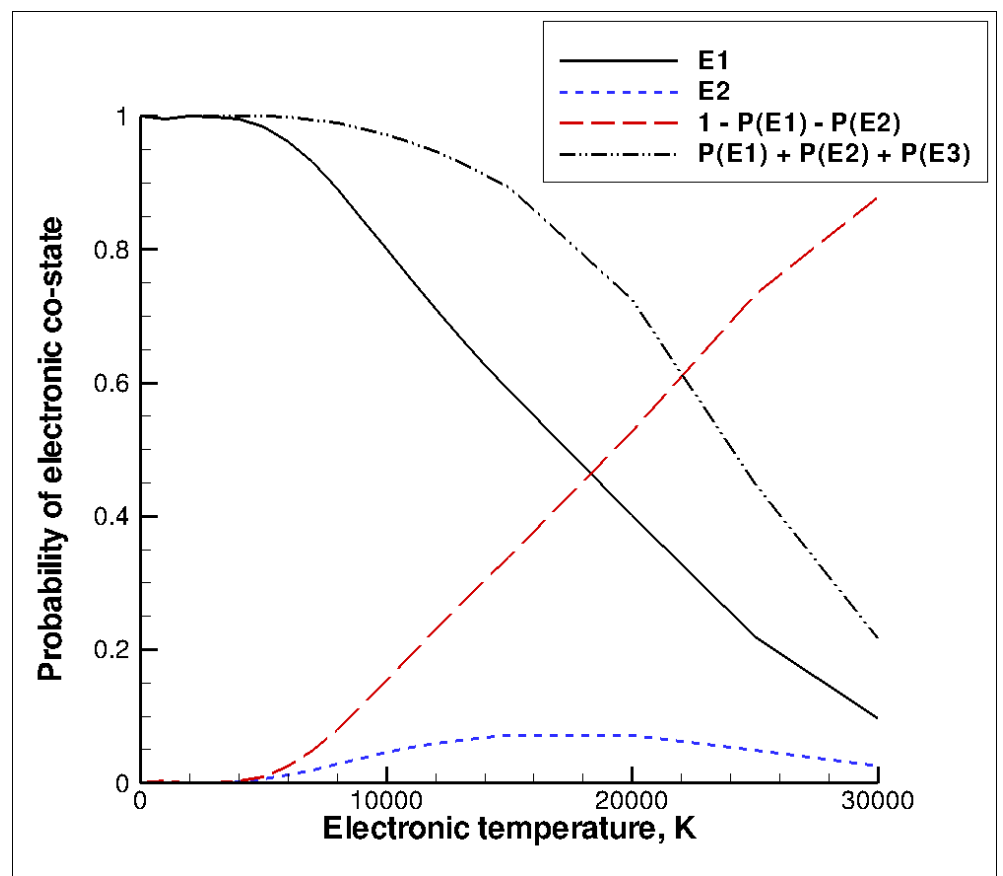

Figure 13: Distribution of electronic co-states used to apply cross section data for $\mathrm{N}+\mathrm{O}$ AI reaction. 


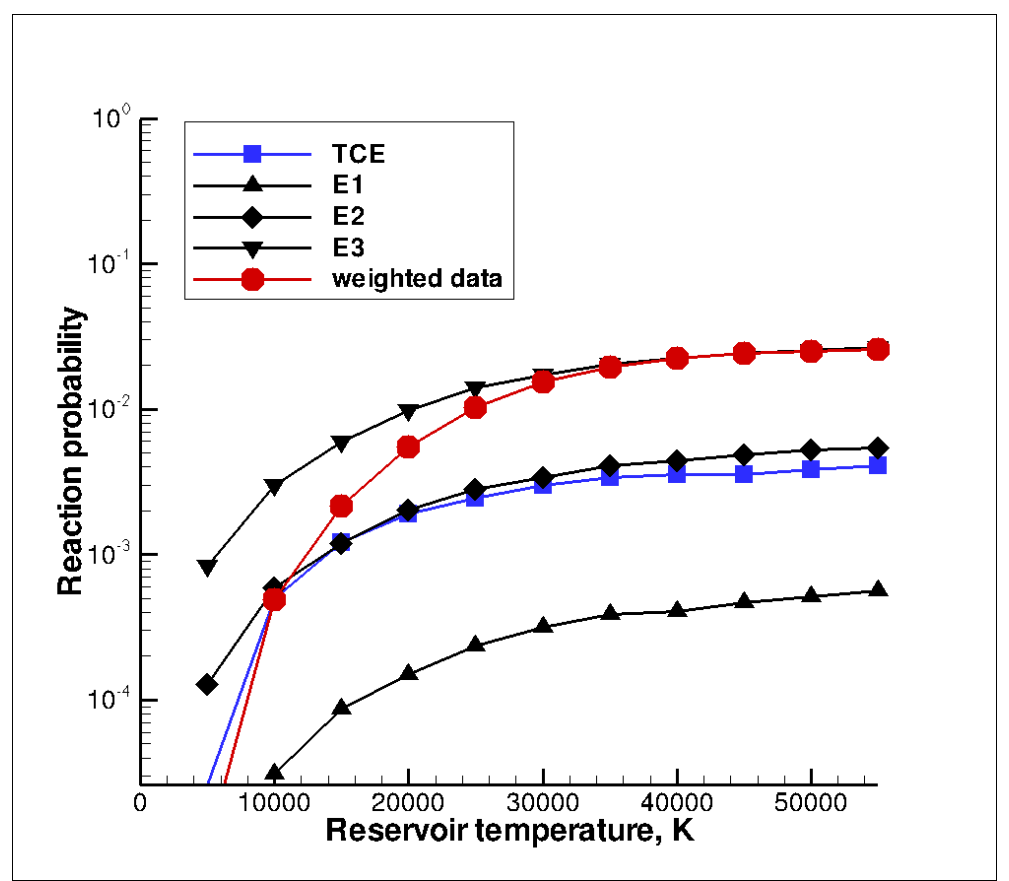

Figure 14: Reaction probabilities for associative ionization of $\mathrm{N}+\mathrm{O}$.

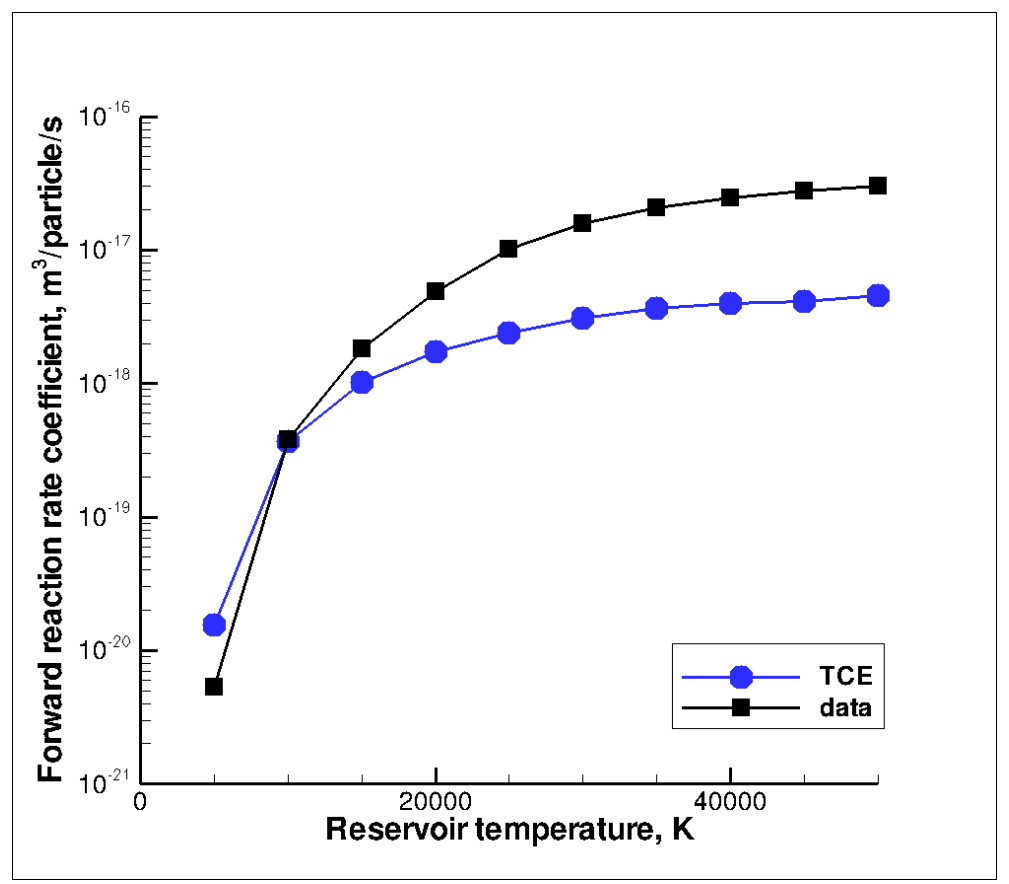

Figure 15: Reaction rate constants for associative ionization of $\mathrm{N}+\mathrm{O}$. 


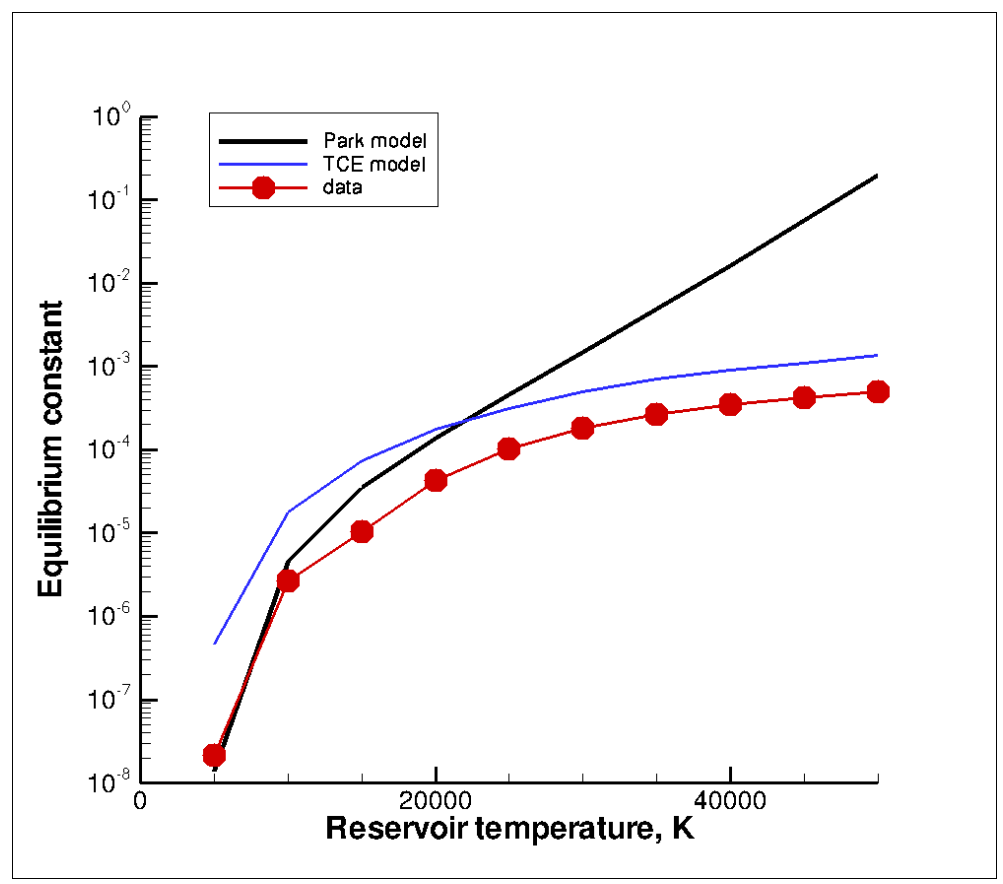

Figure 16: Equilibrium constants for $\mathrm{N}+\mathrm{O} \leftrightarrow \mathrm{NO}^{+}+$e.

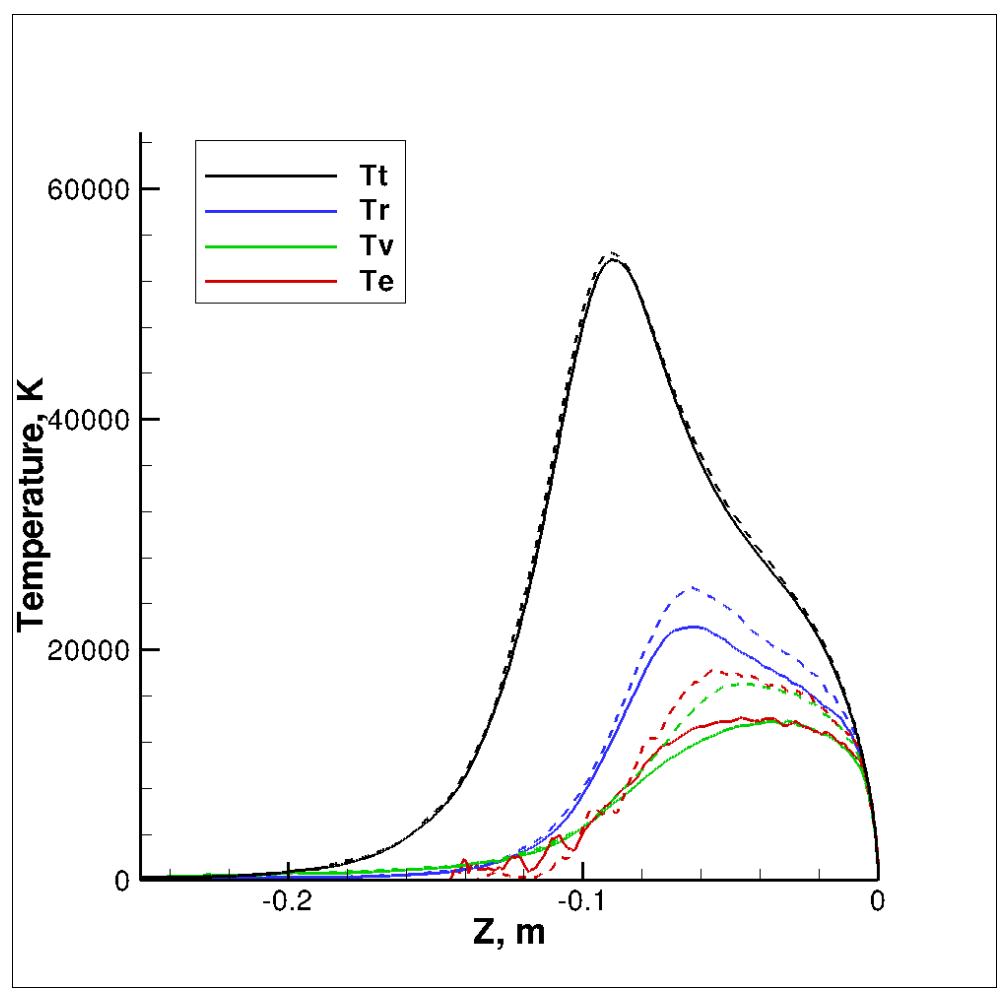

Figure 17: Comparison of the mode temperatures along the stagnation line for FIRE II at 1631s computed using the TCE model to those computed using the cross section data for electron impact dissociation of $\mathrm{N}_{2}$. Solid lines correspond to TCE model, dashed lines correspond to cross section data. 


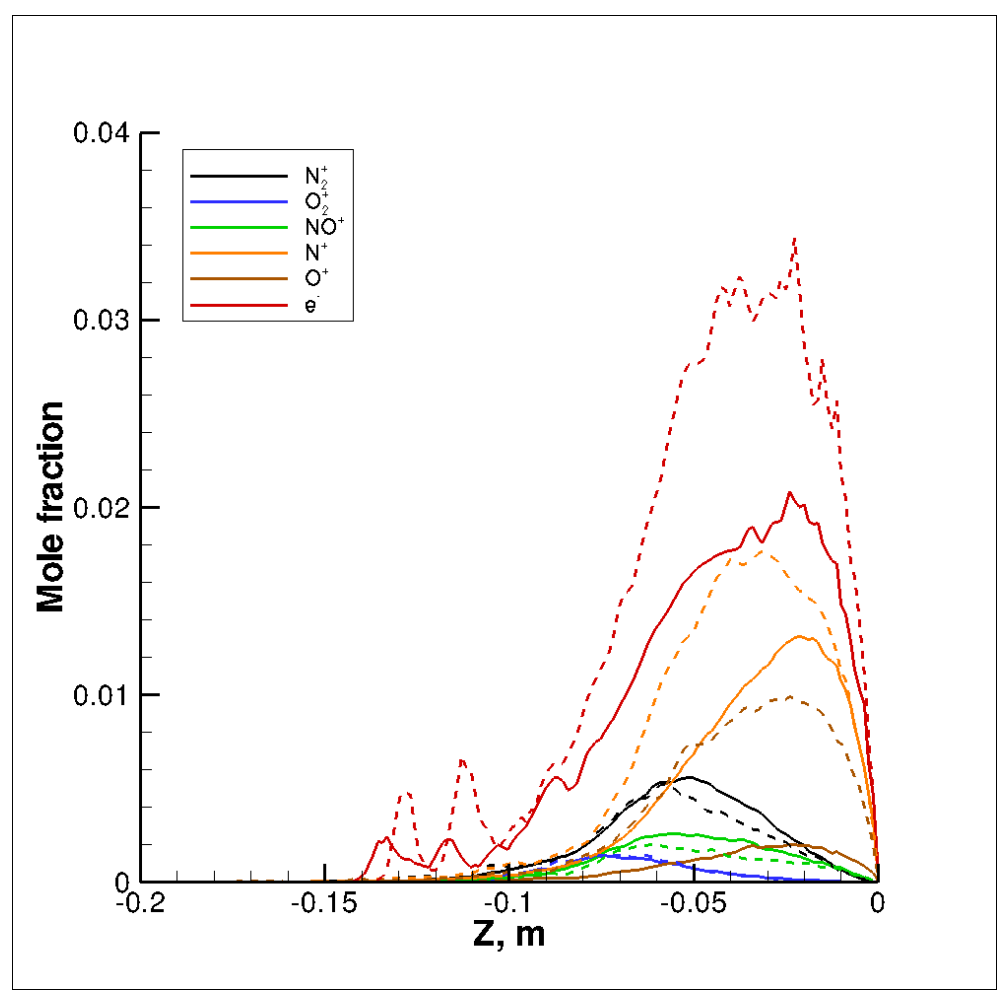

Figure 18: Comparison of the mass fractions of charged species along the stagnation line for FIRE II at 1631s computed using the TCE model to those computed using the cross section data for electron impact dissociation of $\mathrm{N}_{2}$. Solid lines correspond to TCE model, dashed lines correspond to cross section data. 


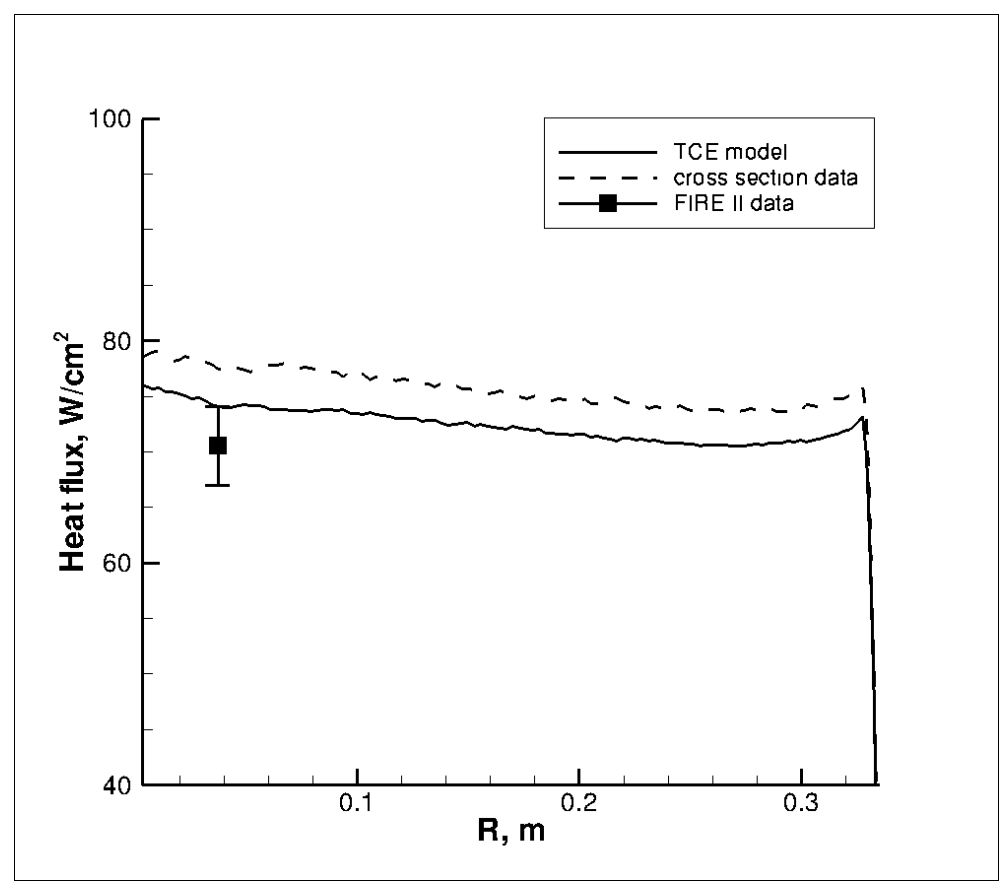

Figure 19: Comparison of the heat flux at the capsule surface for FIRE II at 1631s computed using the TCE model to that computed using the cross section data for electron impact dissociation of $\mathrm{N}_{2}$. Solid lines correspond to TCE model, dashed lines correspond to cross section data.

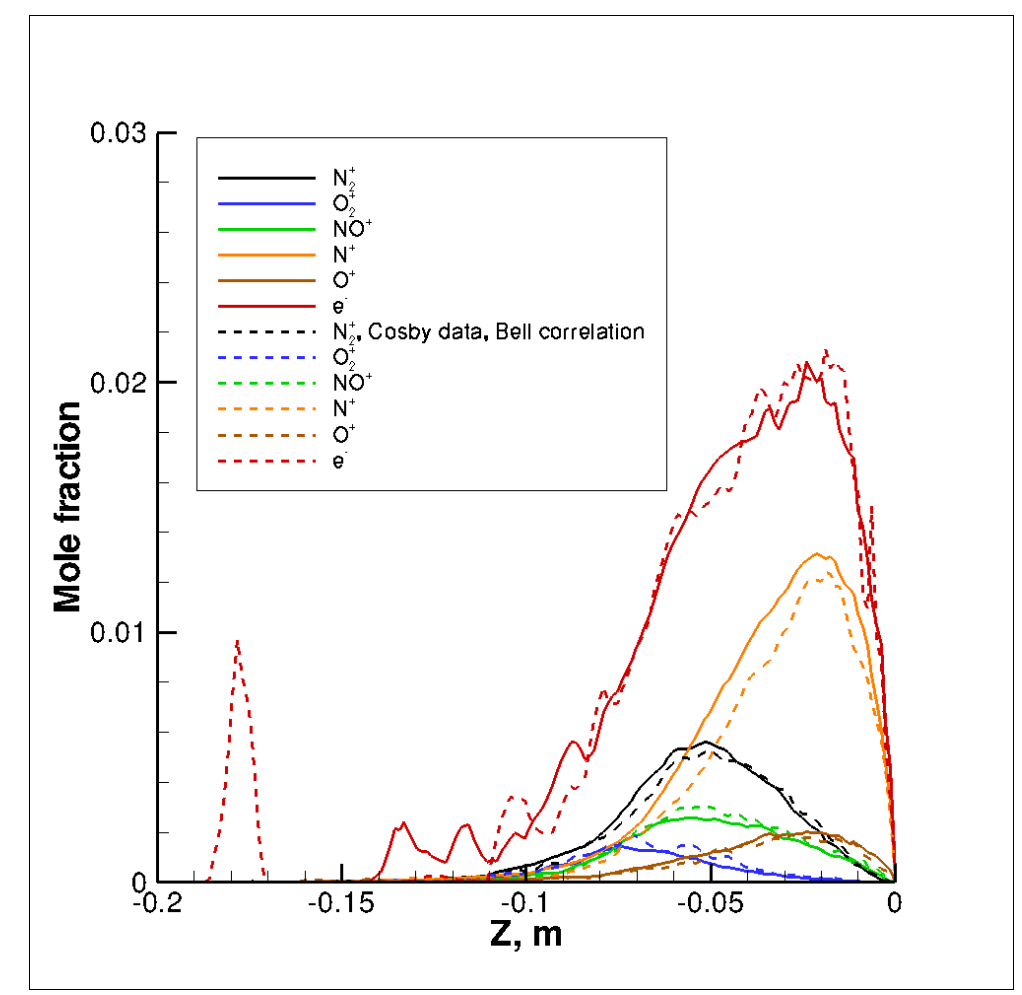

Figure 20: Comparison of the mass fractions of charged species along the stagnation line for FIRE II at 1631s computed using the TCE model to those computed using both the data for electron impact dissociation of $\mathrm{N}_{2}$ and electron impact ionization of $\mathrm{N}$ and O. Solid lines correspond to TCE model, dashed lines correspond to cross section data. 


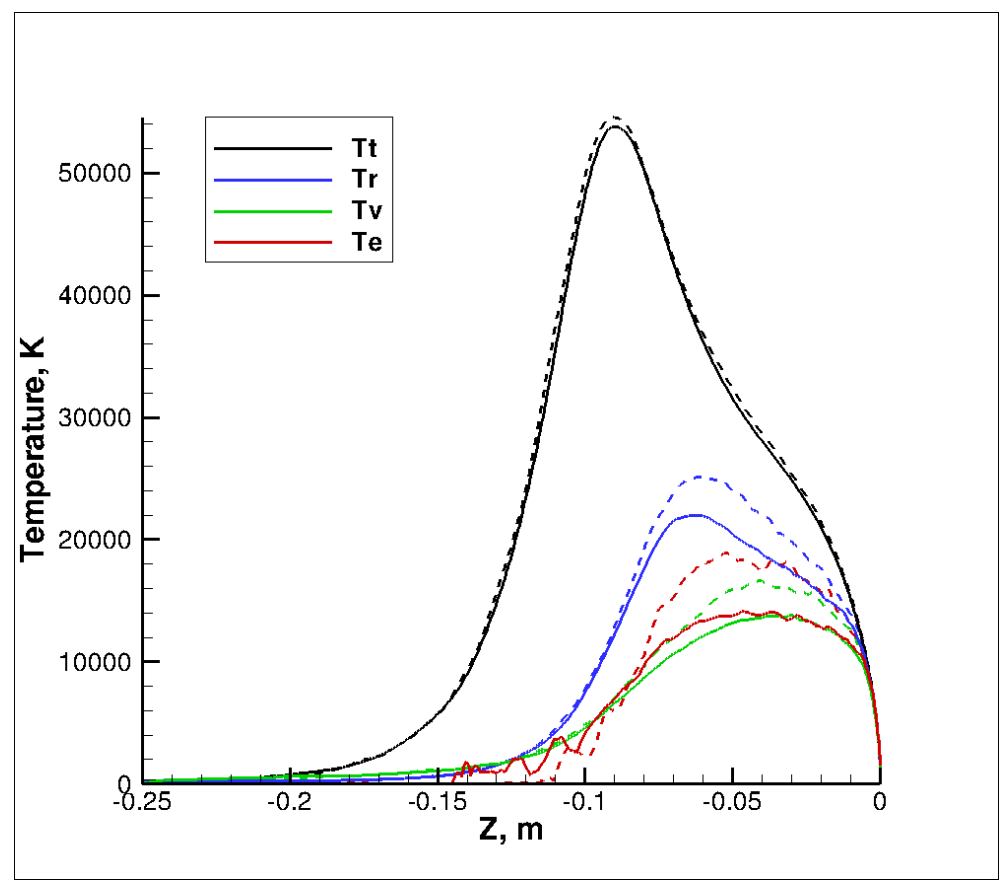

Figure 21: Comparison of the mode temperatures along the stagnation line for FIRE II at 1631s computed using the TCE model to those computed using both the data for electron impact dissociation of $\mathrm{N}_{2}$ and electron impact ionization of $\mathrm{N}$ and $\mathrm{O}$. Solid lines correspond to TCE model, dashed lines correspond to cross section data.

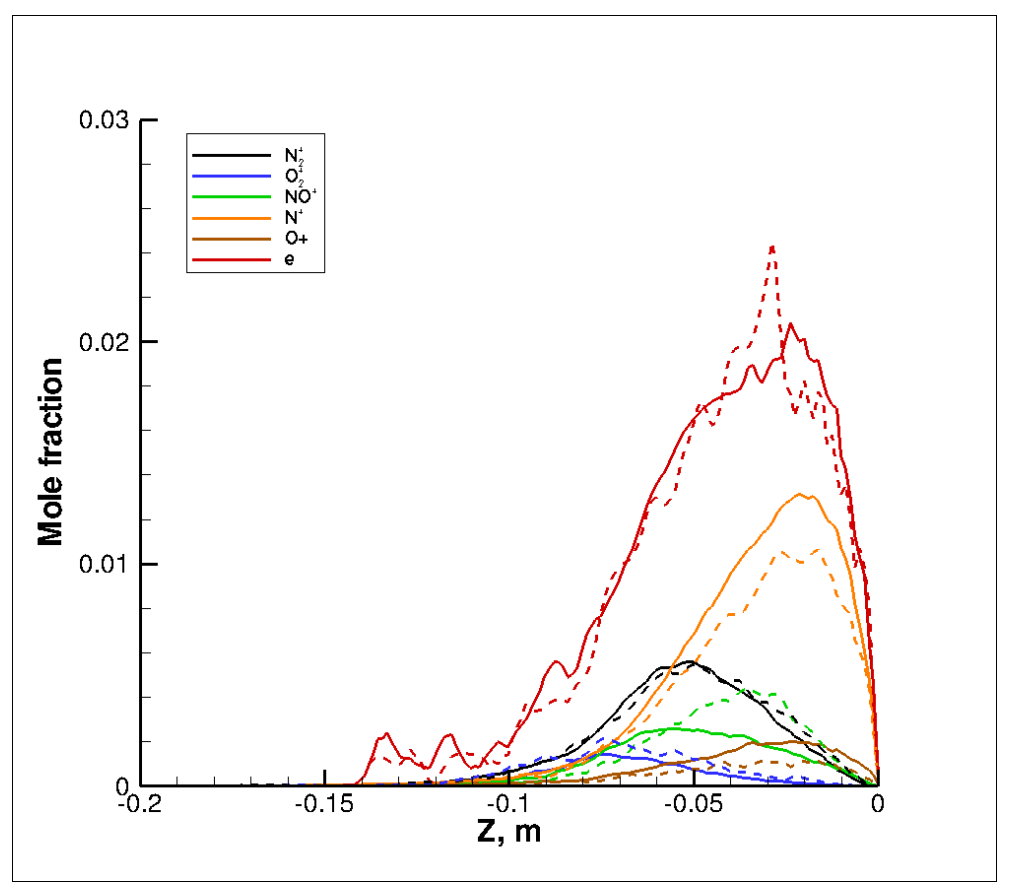

Figure 22: Comparison of the mass fractions of charged species along the stagnation line for FIRE II at 1631s computed using the TCE model to those computed using the data for associative ionization of $\mathrm{N}+$ O. Solid lines correspond to TCE model, dashed lines correspond to cross section data. 